\title{
VARIATIONAL METHODS FOR BIEBERBACH-EILENBERG FUNCTIONS AND FOR PAIRS
}

\author{
J. A. HUMMEL and M. M. SCHIFFER*
}

\section{Introduction}

1.1. We let $U$ denote the unit disc, $|z|<1$, and $\mathscr{S}$ the usual class of univalent functions, i.e., those functions $f(z)$ which are analytic and univalent in $U$ with $f(0)=0, f^{\prime}(0)=1$. A function $F(z)$ is called a Bieberbach-Eilenberg function if it is analytic in $U$, zero at $z=0$, so that it has a series development of the form

$$
F(z)=b_{1} z+b_{2} z^{2}+\ldots,
$$

and is such that

$$
F\left(z_{1}\right) F\left(z_{2}\right) \neq 1
$$

for any $z_{1}, z_{2} \in U$. In this paper we will be concerned only with the subclass of these functions which are univalent in $U$ and we will denote this class of univalent Bieberbach-Eilenberg functions by $\mathscr{E}$.

Two functions, $F$ and $G$, are called $a$ pair if they are analytic in $U, F(0)=$ $=G(0)=0$, and such that

$$
F\left(z_{1}\right) G\left(z_{2}\right) \neq 1
$$

for any $z_{1}, z_{2} \in U$. We will use the term pair only in this sense in this paper. Note that if $F \in \mathscr{E}$, then $\{F, F\}$ is a univalent pair.

1.2. The class $\mathscr{E}$ was introduced by Bieberbach [7] as an aid in solving the problem of maximizing the diameter of the boundary of the image of the complement of a disc (or equivalently any simply connected domain containing $\infty$ ) under all conformal maps which carry $\infty$ to $\infty$ and have derivative 1 at $\infty$. He showed

* This work was supported in part by NSF grant GP 28970 az University of Maryland and by NSF grant MPS72-04967A02 at Stanford University.

doi:10.5186/aasfm.1977.0310 
that the extremal function maps onto the exterior of a line segment by proving that if $F \in \mathscr{E}$, then

$$
\left|b_{1}\right| \leqq 1,
$$

with equality if and only if $F(z)=e^{i \alpha} z, \alpha$ real. The crucial part of his argument was the observation that the function $w+1 / w$ has a single valued inverse in the exterior of a continuum passing through \pm 2 . It therefore defines a univalent mapping from any domain in the $w$-plane having the property (1.2) onto the exterior of such a continuum.

Eilenberg [9] introduced the full class of (not necessarily univalent) functions satisfying (1.1) and (1.2). He showed that (1.4) held in this class with the help of a topological theorem and the subordination principle. Our use of $\mathscr{E}$ to denote the univalent class is therefore somewhat misleading, but we do not want to cause confusion with the standard class of bounded functions.

Rogosinski [28] simplified Eilenberg's results considerably and extended the subordination principle. Using his methods as found in [29], many results obtained in $\mathscr{E}$ can be extended to the full class. Some care is required however. See for example the discussion in $\S 9$ of [20].

In [28], Rogosinski conjectured that all $\left|b_{n}\right| \leqq 1$. This was proved by Lebedev and Milin [23], later Aharonov [3] and Nehari [25] independently proved that $\left|b_{n}\right| \leqq$ $\leqq e^{-\gamma / 2} / \sqrt{n-1}$ for all $n$; where $\gamma$ is the Euler constant.

1.3. Many major results concerning Bieberbach-Eilenberg functions were proved by Jenkins in a series of three papers [18, 19, 20]. He studied several extremal problems by considering appropriate module problems and using symmetrization. This allowed him to obtain an explicit upper bound for $|F(r)|$ for $F \in \mathscr{E}$ and $r$ fixed, and implicit solutions of several other problems. He also used area methods in [20] to prove some inequalities of Grunsky type.

1.4. The class $\mathscr{E}$ appeared in an unexpected fashion when Garabedian and Schiffer made a systematic search for extremal problems in $\mathscr{S}$ which would have a simple solution by variational methods. This search is described in [33]. This led to a class of inequalities of Grunsky type which in turn led to a proof of the local Bieberbach conjecture [11]. Some of the inequalities they found could be interpreted as inequalities for functions in $\mathscr{E}$, because of a close connection between $\mathscr{S}$ and $\mathscr{E}$.

If $f(z) \in \mathscr{S}$, with $1 / u \notin f(U)$, then

$$
F(z)=\frac{1-[1-u f(z)]^{1 / 2}}{1+[1-u f(z)]^{1 / 2}}
$$

is in $\mathscr{E}$. This can be inverted, giving $f(z)=F(z) /\left\{F^{\prime}(0)[1+F(z)]^{2}\right\}, F^{\prime}(0)=u / 4$, showing how $F$ near the identity in $\mathscr{E}$ corresponds to $f$ near the Koebe function. It was this fact which was important in the proof of the local Bieberbach conjecture in [11] and which indicates the potential importance of studying the class $\mathscr{E}$. 
1.5. The concept of pairs was introduced by Aharonov in 1969 in [1]. In some respects, this concept is equivalent to considering functions $f$ and $g$ which have disjoint ranges with $f(=F)$ analytic and $g(=1 / G)$ meromorphic in $U$. Functions with disjoint ranges had been studied by several authors earlier, for example $[5,6$, $14,20,22]$. In particular, see the supplements to [12]. However, Aharonov's class seems to be the correct one to consider as a generalization of Bieberbach-Eilenberg functions, as is evident from some of the results of the present paper.

The main previously known results about pairs are found in $[1,2,16,21]$. Some of these are discussed in the following sections.

1.6. Variational methods are powerful tools in the study of univalent functions. They offer one of the most systematic and widely applicable methods of attacking extremal problems. The variational method in the class $\mathscr{S}$ introduced in [31] has been generalized considerably, and systematic methods of using this and similar variations have been found. See for example the discussion in [32].

The development of variational methods for special classes depends on finding a method of varying the image domain so as to preserve the desired property. In particular, it does not seem immediately obvious how to do this in the case of the class $\mathscr{E}$.

D. J. Nelson, in his thesis [26], obtained a variation in the class $\mathscr{E}$ by using (1.5) to map $F \in \mathscr{E}$ to $f \in \mathscr{S}$. The well-known variation [31] of $\mathscr{S}$ was used on $f$ and the resulting varied function was mapped back to $\mathscr{E}$ by (1.5) again. The extra parameter $u$ which had to be introduced could be eliminated so that he was able to obtain the differential equation satisfied by the extremal function for some specific problems. Some of the observations he made for these problems are generalized and appear in Theorem 4.2 below. In particular, Nelson obtained the differential equation (4.3) for the $F \in \mathscr{E}$ maximizing $\operatorname{Re} b_{n}$, and studied the specific problem of maximizing $\operatorname{Re} b_{2}$, obtaining some bounds on the maximum value.

1.7. In Sections 2 and 3 of this paper, we obtain variational methods for the class $\mathscr{E}$ and for univalent pairs. In Sections 4 and 5 we show how to apply these variations to quite general types of extremal problems. The remainder of the paper is devoted to studying a representative set of extremal problems.

An extremal function for such a problem is found to satisfy a differential equation of the form $Q(w) d w^{2}=R(z) d z^{2}$ where $Q$ and $R$ are typically rational functions which unfortunately involve unknown parameters. Each side of this equation is a quadratic differential, and we obtain information about the solution by considering the trajectories of these quadratic differentials. A simple discussion of the structure of such trajectories may be found, for example, in [4]. Here we will review some elementary facts about such trajectories.

A trajectory of the quadratic differential $d \Omega^{2}=Q(w) d w^{2}$ where $Q(w)$ is a rational function is a path $w=w(t), a<t<b$ along which $d \Omega^{2}>0$. An orthogonal trajectory is a path on which $d \Omega^{2}<0$. A critical point is a point at which $Q(w)$ 
has a zero or pole. If $w_{0}$ is not a critical point, then $\Omega=\int \sqrt{Q(w)} d w$ is analytic and univalent in a neighborhood of $w_{0}$ and hence there exists a unique trajectory through $w_{0}$ (and similarly a unique orthogonal trajectory). Two trajectories can meet only at a critical point. A trajectory can be continued indefinitely unless it closes or reaches a critical point.

The structure of trajectories near a critical point can be complicated, but we will need only two special cases in this paper. If $Q(w)$ has a simple pole at $w_{0}$, then exactly one trajectory of $d \Omega^{2}$ leaves $w_{0}$. If $Q(w)$ has a simple zero at $w_{1}$, then three trajectories leave $w_{1}$. These make equal angles with one another.

\section{A method of variation for Bieberbach-Eilenberg functions}

2.1. To use the known formulas for the variation of the Green's function to obtain a variation of the mapping function, we must have a method of varying a domain so as to stay within the desired class. Define a Bieberbach-Eilenberg domain to be the image of a function in $\mathscr{E}$, that is, a simply connected domain containing the origin which is such that if $w$ is in the domain, then $1 / w$ is not.

Theorem 2.1. Let $D$ be a Bieberbach-Eilenberg domain. Let $\Delta$ be a domain whose closure does not contain 0 or $\infty$, which contains the boundary of $D$, and which is symmetric with respect to the mapping $w \rightarrow 1 / w$ (i.e., $w \in \Delta$ iff $1 / w \in \Delta$ ). Let $\Phi(w)$ be analytic in $\bar{\Delta}$ and satisfy

$$
\Phi(w)=-\Phi(1 / w)
$$

for all $w \in \Delta$.

Then for all \& sufficiently near 0 , the function

$$
w^{*}(w)=w e^{\varepsilon \Phi(w)}
$$

is univalent in $\Delta$ and maps the boundary of $D$ onto the boundary of a BieberbachEilenberg domain.

Proof. Introduce the function

$$
\begin{aligned}
\Psi(w, \omega) & =\frac{\Phi(w)-\Phi(\omega)}{w-\omega} & & w \neq \omega \\
& =\Phi^{\prime}(w) & w & =\omega .
\end{aligned}
$$

This $\Psi$ is defined, analytic, and uniformly bounded in the compact set $\bar{\Delta} \times \bar{\Delta}$.

Suppose $w^{*}(w)$ were not univalent in $\Delta$. Then there would exist $w_{1} \neq w_{2}$ in $\triangle$ such that $w^{*}\left(w_{1}\right)=w^{*}\left(w_{2}\right)$. That is, $w_{1}-w_{2}=w_{1}\left[1-\exp \left\{\varepsilon\left(w_{1}-w_{2}\right) \Psi\left(w_{1}, w_{2}\right)\right\}\right]$. Now for any $s,\left|1-e^{s}\right| \leqq|s| e^{|s|}$, so this implies

$$
\left|w_{1}-w_{2}\right| \leqq|\varepsilon| \cdot\left|w_{1}\left(w_{1}-w_{2}\right) \Psi\left(w_{1}, w_{2}\right)\right| \exp \left\{\left|\varepsilon\left(w_{1}-w_{2}\right) \Psi\left(w_{1}, w_{2}\right)\right|\right\} .
$$

Divide through by $\left|w_{1}-w_{2}\right|$. The stated conditions on $\Delta$ then give a contradiction for all sufficiently small $|\varepsilon|$. 
Since $w^{*}(w)$ is univalent in $\Delta$, it maps the boundary of $D$ onto the boundary of a simply connected domain $D^{*}$. It remains to show that $D^{*}$ is a BieberbachEilenberg domain for all sufficiently small $|\varepsilon|$.

This property is equivalent to asserting that $D^{*}$ and $D_{1}^{*}=\left\{w: 1 / w \in D^{*}\right\}$ do not intersect. Suppose to the contrary that they do. Then they must in fact intersect at points arbitrarily close to the boundary of $D^{*}$ and we can therefore assume that there exist $w_{1}$ and $w_{2}$ in $\Delta \cap D$ such that $w^{*}\left(w_{1}\right) w^{*}\left(w_{2}\right)=1$, i.e., that $w_{1} w_{2} \exp \left\{\varepsilon\left[\Phi\left(w_{1}\right)+\Phi\left(w_{2}\right)\right]\right\}=1$. Using (2.1) this is equivalent to

$$
\left(w_{1}-w_{2}^{-1}\right)=w_{1}\left[1-\exp \left\{\varepsilon\left(w_{1}-w_{2}^{-1}\right) \Psi\left(w_{1}, w_{2}^{-1}\right)\right\}\right] .
$$

Since $w_{1}, w_{2} \in D, w_{1} \neq w_{2}^{-1}$, and we may proceed exactly as before to obtain a contradiction as $|\varepsilon| \rightarrow 0$. This proves the theorem.

2.2. We use this theorem to find a variational formula for functions in $\mathscr{E}$ using the variational formula for the Green's function [31]. The form we use is described in [32]. Let $D$ be a domain and $D^{*}$ a varied domain obtained from $D$ by the mapping $w^{*}=w+\varepsilon v(w)+o(\varepsilon)$ defined and univalent near the boundary in $D$. If $p(w, \omega)$ and $p^{*}(w, \omega)$ are the analytic completions of the Green's functions of $D$ and $D^{*}$, then

$$
p^{*}(w, \omega)=p(w, \omega)+\varepsilon q_{1}(w, \omega)+o(\varepsilon)
$$

where $q_{1}(w, \omega)$ is an analytic function of $w$ such that

$$
\operatorname{Re}\left\{q_{1}(w, \omega)\right\}=\operatorname{Re}\left\{\frac{1}{2 \pi i} \int_{\Gamma} p^{\prime}(t, w) p^{\prime}(t, \omega) v(t) d t\right\}
$$

and $\Gamma$ is a curve system in $D$ bounding a subdomain of $D$ containing $w$ and $\omega$.

Let $F$ map $U$ onto $D$ and $F^{*}$ map $U$ onto $D^{*}$ with $F(0)=F^{*}(0)=0$. If $\varphi$ and $\varphi^{*}$ are the inverse functions of $F$ and $F^{*}$, then $p(w, 0)=-\log \varphi(w), p^{*}(w, 0)=$ $=-\log \varphi^{*}(w)$ and hence $\varphi^{*}(w)=\varphi(w)-\varepsilon \varphi(w) q_{1}(w, 0)+o(\varepsilon)$. Then, as in [32], it follows that

$$
F^{*}(z)=F(z)+\varepsilon z F^{\prime}(z) q_{1}(F(z), 0)+o(\varepsilon) .
$$

Notice that the derivative at 0 is not normalized in this class.

Let $w_{0} \in D$ and let $\alpha$ be any real number. Set

$$
\Phi(w)=e^{i \alpha}\left[\frac{1}{w-w_{0}}-\frac{w}{1-w_{0} w}\right] .
$$

This $\Phi$ satisfies (2.1). The remaining hypotheses of Theorem 2.1 hold and hence the variation $w^{*}=w e^{\varepsilon \Phi(w)}=w+\varepsilon w \Phi(w)+o(\varepsilon)$ preserves the class. We need only compute $q_{1}(w, 0)$ using $v(w)=w \Phi(w)$.

2.3. Let $\varphi$ be the inverse function of $F$. Then

$$
p(t, w)=-\log ([\varphi(t)-\varphi(w)] /[1-\overline{\varphi(w)} \varphi(t)])
$$


and hence from (2.3) we have

$$
\begin{aligned}
& \operatorname{Re}\left\{q_{1}(w, 0)\right\} \\
= & -\operatorname{Re}\left\{\frac{e^{i \alpha}}{2 \pi i} \int_{\Gamma}\left[\frac{\varphi^{\prime}(t)}{\varphi(w)-\varphi(t)}-\frac{\overline{\varphi(w)} \varphi^{\prime}(t)}{1-\overline{\varphi(w)} \varphi(t)}\right] \cdot\left[\frac{\varphi^{\prime}(t)}{\varphi(t)}\right] \cdot\left[\frac{t}{t-w_{0}}-\frac{t^{2}}{1-w_{0} t}\right] d t\right\} \\
= & \operatorname{Re}\left\{e^{i \alpha} \frac{\varphi^{\prime}(w)}{\varphi(w)}\left[\frac{w}{w-w_{0}}-\frac{w^{2}}{1-w_{0} w}\right]-e^{i \alpha} \frac{w_{0} \varphi^{\prime}\left(w_{0}\right)^{2}}{\varphi\left(w_{0}\right)\left[\varphi(w)-\varphi\left(w_{0}\right)\right]}+\right. \\
+ & \left.e^{i \alpha} \frac{w_{0} \varphi^{\prime}\left(w_{0}\right)^{2} \overline{\varphi(w)}}{\varphi\left(w_{0}\right)\left[1-\overline{\varphi(w)} \varphi\left(w_{0}\right)\right]}\right\} .
\end{aligned}
$$

Here we calculated the residues at $t=w$ and $t=w_{0}$. There is no singularity at $t=0$.

We may conjugate the final term inside the real part. The resulting function is analytic in $w$ and may be identified with $q_{1}(w, 0)$. Replacing $w$ by $F(z), w_{0}$ by $F\left(z_{0}\right)$ and so on, (2.4) gives

Theorem 2.2. Let $F(z) \in \mathscr{E}$, let $\alpha$ be any real number, and let $z_{0} \in U$. Then for all sufficiently small $\varepsilon>0$ there exists a function $F^{*}(z) \in \mathscr{E}$ such that

$$
\begin{aligned}
F^{*}(z) & =F(z)+\varepsilon e^{i \alpha}\left[\frac{F(z)}{F(z)-F\left(z_{0}\right)}-\frac{F(z)^{2}}{1-F\left(z_{0}\right) F(z)}\right]-\varepsilon e^{i \alpha}\left(\frac{F\left(z_{0}\right)}{z_{0} F^{\prime}\left(z_{0}\right)^{2}}\right) \frac{z F^{\prime}(z)}{z-z_{0}} \\
& +\varepsilon e^{-i \alpha} \overline{\left(\frac{F\left(z_{0}\right)}{z_{0} F^{\prime}\left(z_{0}\right)^{2}}\right)} \frac{z^{2} F^{\prime}(z)}{1-\bar{z}_{0} z}+o(\varepsilon) .
\end{aligned}
$$

In the calculation of $q_{1}(w, 0)$ we found a residue at $w_{0}$ since $w_{0}$ was assumed in $D$. There was none at $1 / w_{0}$ since $D$ was a Bieberbach-Eilenberg domain. Suppose neither $w_{0}$ nor $1 / w_{0}$ were in $D$. Then the only residue would be at $w$. Thus we have

Theorem 2.3. Let $F(z) \in \mathscr{E}$. Let $\alpha$ be any real number. Suppose $w_{0}$ is such that neither $w_{0}$ nor $1 / w_{0}$ is in the closure of $F(U)$. Then for all sufficiently small $\varepsilon>0$ there exists a function $F^{*}(z) \in \mathscr{E}$ such that

$$
F^{*}(z)=F(z)+\varepsilon e^{i \alpha}\left[\frac{F(z)}{F(z)-w_{0}}-\frac{F(z)^{2}}{1-w_{0} F(z)}\right]+o(\varepsilon) .
$$

\section{A method of variation for pairs}

3.1. Let $\left\{F_{1}(z), F_{2}(z)\right\}$ be a univalent pair. Set $D_{1}=F_{1}(U), D_{2}=F_{2}(U), D_{2}^{\prime}=$ $=\left\{w: 1 / w \in D_{2}\right\}$. Suppose $\Delta_{0}$ is an open set which contains the boundaries of $D_{1}$ and $D_{2}^{\prime}$, and that $\Phi(w)$ is analytic in the closure of $\Delta_{0}$. Then one easily verifies that

$$
w^{*}(w)=w+\varepsilon \Phi(w)
$$


is univalent in $\Delta_{0}$ for all sufficiently small $\varepsilon$. This will induce a variation of $D_{1}$ to $D_{1}^{*}$ and hence of $F_{1}$ to $F_{1}^{*}$. At the same time (3.1) induces a variation of $D_{2}^{\prime}$ to $D_{2}^{* \prime}$ and hence of $D_{2}$ to $D_{2}^{*}$ and of $F_{2}$ to $F_{2}^{*}$ so that $\left\{F_{1}^{*}, F_{2}^{*}\right\}$ will be a new univalent pair.

As in $\S 2.2$, we have

$$
F_{1}^{*}(z)=F_{1}(z)+\varepsilon z F_{1}^{\prime}(z) q_{1}\left(F_{1}(z), 0\right)+o(\varepsilon)
$$

where $q_{1}(w, 0)$ is an analytic function of $w$ satisfying

$$
\operatorname{Re}\left\{q_{1}(w, 0)\right\}=\operatorname{Re}\left\{\frac{1}{2 \pi i} \int_{\Gamma} p_{1}^{\prime}(t, w) p_{1}^{\prime}(t, 0) \Phi(t) d t\right\}
$$

the quantities being defined as in $\S 2.2$.

To find the variation of $D_{2}$ induced by (3.1), let $\omega=1 / w$. Then $\omega^{*}=\omega+\varepsilon \Phi(\omega)$ and hence $w^{*}=1 / \omega^{*}=\omega^{-1}[1+\varepsilon \Phi(\omega) / \omega]^{-1}=\omega^{-1}[1-\varepsilon \Phi(\omega) / \omega]+o(\varepsilon)=w-\varepsilon w^{2} \Phi(1 / w)+$ $+o(\varepsilon)$. Hence

$$
F_{2}^{*}(z)=F_{2}(z)+\varepsilon z F_{2}^{\prime}(z) q_{2}\left(F_{2}(z), 0\right)+o(\varepsilon)
$$

where $q_{2}$ is determined as before by

$$
\operatorname{Re}\left\{q_{2}(w, 0)\right\}=-\operatorname{Re}\left\{\frac{1}{2 \pi i} \int_{\Gamma_{2}} p_{2}^{\prime}(t, w) p_{2}^{\prime}(t, 0) t^{2} \Phi(1 / t) d t\right\} .
$$

3.2. Let $w_{0}$ be any point which is neither on the boundary of $D_{1}$ nor in the closure of $D_{2}^{\prime}$. Let $\alpha$ be real and set

$$
\Phi(w)=\frac{e^{i \alpha} w}{w-w_{0}} .
$$

Then we may let $\Delta_{0}=\left\{w:\left|w-w_{0}\right|>\delta\right\}$ for some sufficiently small $\delta$. Note that

$$
-w^{2} \Phi(1 / w)=-\frac{e^{i \alpha} w^{2}}{1-w_{0} w} .
$$

Together, (3.6) and (3.7) make up the variation used in 2.3. Thus we have split the variation for the class $\mathscr{E}$ into separate variations for each member of the pair.

There are two possibilities for $w_{0}$ in order to satisfy the stated requirements. We may have $w_{0}=F_{1}\left(z_{0}\right)$ for some $z_{0} \in U$, or $w_{0}$ may lie in the interior of the complement of $D_{1} \cup D_{2}^{\prime}$ (if this set has an interior). In the latter case $\Phi(w)$ is analytic in $D_{1}$. In either case $-w^{2} \Phi(1 / w)$ is analytic in $D_{2}$.

Proceeding as in Section 2, one easily calculates the integrals in (3.3) and (3.5), and proves

Theorem 3.1. Let $\left\{F_{1}(z), F_{2}(z)\right\}$ be any univalent pair. Let $\alpha$ be any real number and let $z_{0} \in U$. Then for all sufficiently small $\varepsilon>0$ there exists a univalent pair 
$\left\{F_{1}^{*}(z), F_{2}^{*}(z)\right\}$ such that

$$
\begin{gathered}
F_{1}^{*}(z)=F_{1}(z)+\varepsilon e^{i \alpha} \frac{F_{1}(z)}{F_{1}(z)-F_{1}\left(z_{0}\right)}-\varepsilon e^{i \alpha}\left(\frac{F_{1}\left(z_{0}\right)}{z_{0}} \frac{z F_{1}^{\prime}(z)}{\left.z-z_{0}\right)^{2}}\right) \frac{z^{2} F_{1}^{\prime}(z)}{1-\bar{z}_{0} z}+o(\varepsilon), \\
+\varepsilon e^{-i \alpha} \overline{\left(\frac{F_{1}\left(z_{0}\right)}{z_{0} F_{1}^{\prime}\left(z_{0}\right)^{2}}\right)} \\
F_{2}^{*}(z)=F_{2}(z)-\varepsilon e^{i \alpha} \frac{F_{2}(z)^{2}}{1-F_{1}\left(z_{0}\right) F_{2}(z)}+o(\varepsilon) .
\end{gathered}
$$

If there exists a $w_{0}$ such that $w_{0}$ is not in the closure of $F_{1}(U)$ and $w_{0}^{-1}$ is not in the closure of $F_{2}(U)$, then for all real $\alpha$ and all sufficiently small $\varepsilon>0$ there exists a univalent pair $\left\{F_{1}^{*}(z), F_{2}^{*}(z)\right\}$ such that

$$
\begin{aligned}
& F_{1}^{*}(z)=F_{1}(z)+\varepsilon e^{i \alpha} \frac{F_{1}(z)}{F_{1}(z)-w_{0}}+o(\varepsilon) \\
& F_{2}^{*}(z)=F_{2}(z)-\varepsilon e^{i \alpha} \frac{F_{2}(z)^{2}}{1-w_{0} F_{2}(z)}+o(\varepsilon) .
\end{aligned}
$$

We remark that $\left\{F_{1}, F_{2}\right\}$ is a univalent pair if and only if $\left\{F_{2}, F_{1}\right\}$ is also. Hence the roles of $F_{1}$ and $F_{2}$ may be reversed in the above theorem.

\section{General extremal problems in the class $\mathscr{E}$}

4.1. The usual class $\mathscr{S}$ of univalent functions is normalized by the requirement that $f(0)=0$ and $f^{\prime}(0)=1$ for any $f \in \mathscr{S}$. Similar normalizations are impossible in $\mathscr{E}$ since the addition of a constant or multiplication by a constant may spoil the Bieberbach-Eilenberg property of a domain. Indeed, there are only two elementary transformations of the image domain which are available in the class. If $F(z) \in \mathscr{E}$, then so are $-F(z)$ and $\overline{F(\bar{z})}$. These correspond to symmetries of the BieberbachEilenberg property and are often useful.

Transformations can however be made freely in the $z$-plane. If $g(z)$ is univalent in $U, g(0)=0$, and $g(U) \subset U$, then $F(z) \in \mathscr{E}$ implies $F(g(z)) \in \mathscr{E}$. In particular, $F\left(e^{i \alpha} z\right) \in \mathscr{E}$ for any real $\alpha$. Thus we can assume $F^{\prime}(0)>0$ if this seems like a useful normalization in a particular problem.

By letting $\alpha$ be near zero, this last transformation can be viewed as a variation in the class $\mathscr{E}$. That is, if $F(z) \in \mathscr{E}$, then so is $F^{*}(z)$ where

$$
F^{*}(z)=F\left(e^{i \varepsilon} z\right)=F(z)+i \varepsilon z F^{\prime}(z)+o(\varepsilon)
$$

for real $\varepsilon$.

Another useful variation is the slit variation obtained by letting $g(z)$ map $U$ onto $U$ less a short slit. Let $k_{\alpha}(z)=z /\left(1+e^{-i \alpha} z\right)^{2}$ be the Koebe function mapping $U$ onto the exterior of the radial slit from $e^{i \alpha} / 4$ to $\infty$. Let $\varepsilon>0$, and define $g(z)$ 
by $k_{\alpha}(g(z))=k_{\alpha}(z) /(1+\varepsilon)$. This is equivalent to $(1+\varepsilon)\left(z^{-1}+2 e^{-i \alpha}+e^{-2 i \alpha} z\right)=$ $\left(1 / g+2 e^{-i \alpha}+e^{-2 i \alpha} g\right)$ and hence $g(z)=z+\varepsilon g_{\alpha}(z)+o(\varepsilon)$ where

$$
g_{\alpha}(z)=-\frac{z\left(1+e^{-i \alpha} z\right)}{1-e^{-i \alpha} z} .
$$

Thus, if $F \in \mathscr{E}$ and $\alpha$ is any real number, $F^{*}(z) \in \mathscr{E}$ also where

$$
F^{*}(z)=F(g(z))=F(z)-\varepsilon z F^{\prime}(z) \frac{e^{i \alpha}+z}{e^{i \alpha}-z}+o(\varepsilon)
$$

and $\varepsilon>0$. These two well-known variations will be usefull in what follows.

4.2. In considering extremal problems in $\mathscr{E}$, one must be careful since the class $\mathscr{E}$ is not compact. One easily sees that any sequence of functions in $\mathscr{E}$ has a subsequence which converges either to a function in $\mathscr{E}$ or to the constant zero. The functions $\{z / n\}_{n=1}^{\infty}$ show that the last possibility can occur. Often, the fact that no extremal function exists in $\mathscr{E}$ is not obvious. For example, if we wish to maximize $\left|b_{2} / b_{1}\right|$ where $F(z)=b_{1} z+b_{2} z^{2}+\ldots$, we find that no maximum exists. The functions mapping $U$ onto $U$ slit from $-1 / n$ to -1 will give $\left|b_{2} / b_{1}\right| \rightarrow 2$, but the uniqueness of the Koebe function maximizing $a_{2}$ in $\mathscr{S}$ shows there is no $F \in \mathscr{E}$ with $\left|b_{2} / b_{1}\right|=2$.

In most of the problems we consider, the existence of an extremal function will be obvious. For example, consider the maximum absolute value of $b_{n}$, the $n$-th coefficient of $F \in \mathscr{E}$. We can always assume $b_{n}>0$ and so look for $F$ maximizing $\operatorname{Re} b_{n}$. A sequence of functions whose $n$-th coefficient converged to the supremum of such values could not tend to zero, and hence the extremal $F$ must exist. It is instructive to see what the variation of Theorem 2.2 implies in this case.

For every $\alpha$ and $z_{0}$ we must have $\operatorname{Re} b_{n}^{*} \leqq \operatorname{Re} b_{n}$ for the extremal $F$. Since the real part is unchanged if we conjugate the term containing $e^{-i \alpha}$ in (2.6), the terms of order $\varepsilon$ must have an $n$-th coefficient which vanishes. One easily sees that this implies that any extremal $F$ must satisfy the differential equation

$$
\left(\frac{z F^{\prime}(z)}{F(z)}\right)^{2} \sum_{k=1}^{n-1} b_{n}^{(k+1)}\left(\frac{1}{F(z)^{k}}+F(z)^{k}\right)=\sum_{k=1}^{n-1} \frac{(n-k) b_{n-k}}{z^{k}}+n b_{n}+\sum_{k=1}^{n-1}(n-k) \bar{b}_{n-k} z^{k}
$$

where the $b_{n}^{(k)}$ are defined by $F(z)^{k}=\sum_{n=k}^{\infty} b_{n}^{(k)} z^{n}$.

This is exactly the differential equation which Nelson obtained in his thesis [26] for the same problem.

4.3. We now look at more general extremal problems, following the methods of [32]. Let $\Psi$ be a continuous functional defined over $\mathscr{E}$. We assume that $\Psi$ has a continuous Frêchet (or more generally Gâteaux) derivative $L$. That is, for any $F \in \mathscr{E}$ and any analytic $G$,

$$
\Psi(F+\varepsilon G)=\Psi(F)+\varepsilon L(F ; G)+o(\varepsilon) .
$$


Here $L(F ; G)$ will be a continuous linear functional of $G$ for each fixed $F$. Since $F$ will remain fixed, we will usually suppress the dependence on $F$ and write merely $L(G)$.

Define

$$
\begin{array}{llrl}
D(w) & =L\left(\frac{w F}{F-w}\right) ; & E(\zeta) & =L\left(\frac{\zeta z F^{\prime}(z)}{z-\zeta}\right) \\
A(w) & =D(w)+L(F)+D(1 / w) ; & B(\zeta) & =E(\zeta)+\overline{L\left(z F^{\prime}(z)\right)}+\overline{E(1 / \zeta)} .
\end{array}
$$

Observe that

$$
\begin{aligned}
-\frac{w F^{2}}{1-w F} & =F+\frac{F}{w(F-1 / w)} \\
-\frac{\bar{\zeta} z^{2} F^{\prime}(z)}{1-\bar{\zeta} z} & =z F^{\prime}(z)+\frac{z F^{\prime}(z)}{\bar{\zeta}(z-1 / \bar{\zeta})}
\end{aligned}
$$

and hence the variation of Theorem 2.2 implies

$$
\begin{aligned}
& \Psi\left(F^{*}\right)=\Psi(F)+\varepsilon e^{i \alpha} \frac{1}{F\left(z_{0}\right)} A\left(F\left(z_{0}\right)\right)-\varepsilon e^{i \alpha} \frac{1}{F\left(z_{0}\right)}\left(\frac{F\left(z_{0}\right)}{z_{0} F^{\prime}\left(z_{0}\right)}\right)^{2} E\left(z_{0}\right) \\
& -\varepsilon e^{-i \alpha} \frac{1}{\overline{F\left(z_{0}\right)}} \overline{\left(\frac{F\left(z_{0}\right)}{z_{0} F^{\prime}\left(z_{0}\right)}\right)^{2}}\left[L\left(z F^{\prime}(z)\right)+E\left(1 / \bar{z}_{0}\right)\right]+o(\varepsilon)
\end{aligned}
$$

or

$$
\operatorname{Re} \Psi\left(F^{*}\right)=\operatorname{Re} \Psi(F)+\varepsilon \operatorname{Re} \frac{e^{i \alpha}}{F\left(z_{0}\right)}\left\{A\left(F\left(z_{0}\right)\right)-\left(\frac{F\left(z_{0}\right)}{z_{0} F^{\prime}\left(z_{0}\right)}\right)^{2} B\left(z_{0}\right)\right\}+o(\varepsilon) .
$$

We are interested in problems of the type: maximize $\operatorname{Re} \Psi(F)$ among all $F \in \mathscr{E}$. Such a problem may or may not have a solution, but if it does, we can use (4.7) to characterize this solution. We will say that $F \in \mathscr{E}$ is (locally) extremal for $\operatorname{Re} \Psi(F)$ if $\operatorname{Re} \Psi\left(F^{*}\right) \leqq \operatorname{Re} \Psi(F)$ for all (nearby) $F^{*} \in \mathscr{E}$. Here, "nearby" is in the sense of convergence on compact subsets of $U$. Using (4.7) we therefore can prove:

Theorem 4.1. Let the functional $\Psi$, defined over $\mathscr{E}$, have a continuous linear Frêchet derivative $L(F ; G)$ as defined in (4.4). Let $A(w)$ and $B(\zeta)$ be defined as in (4.5). Suppose $F \in \mathscr{E}$ is locally extremal for $\operatorname{Re} \Psi(F)$. Then $F$ satisfies the differential equation

$$
\left(\frac{z F^{\prime}(z)}{F(z)}\right)^{2} A(F(z))=B(z), \quad z \in U
$$

or equivalently

$$
A(w) \frac{d w^{2}}{w^{2}}=B(z) \frac{d z^{2}}{z^{2}}, \quad w=F(z), \quad z \in U
$$

Further, $L\left(F ; z F^{\prime}(z)\right)$ is real, $B(z)$ is real and non-positive for $|z|=1$, and if $A(F(z))$ is analytic in some annulus $\varrho<|z|<1$, then $F$ maps $U$ onto a domain whose boundary is made up of analytic arcs (or is an analytic curve) which are trajectories of $A(w) d w^{2} / w^{2}$. 
Proof. The fact that $F$ satisfies the differential equation (4.8) or (4.9) follows immediately from (4.7) since $\alpha$ is arbitrary, and $F$ makes $\operatorname{Re} \Psi$ a local maximum. Using the rotational variation (4.1) gives $\operatorname{Re} \Psi\left(F^{*}\right)=\operatorname{Re} \Psi(F)+\varepsilon \operatorname{Re} i L\left(z F^{\prime}(z)\right)+$ $+o(\varepsilon)$. Since $\varepsilon$ can be positive or negative, $L\left(z F^{\prime}(z)\right)$ must be real. If $|\zeta|=1$, then (4.5) shows $B(\zeta)$ is real. Let $\zeta=e^{i \alpha}$. Then the slit variation (4.2) gives

$$
\operatorname{Re} \Psi\left(F^{*}\right)=\operatorname{Re} \Psi(F)-\varepsilon \operatorname{Re} L\left(z F^{\prime}(z) \frac{\zeta+z}{\zeta-z}\right)+o(\varepsilon) .
$$

Since $\varepsilon>0$, this implies $\operatorname{Re} L\left(z F^{\prime}(z)(\zeta+z) /(\zeta-z)\right) \geqq 0$. However, $|\zeta|=1$ and since $(\zeta+z) /(\zeta-z)=-1-2 \zeta /(z-\zeta)$,

$$
\begin{aligned}
B(\zeta) & =\operatorname{Re} B(\zeta)=L\left(z F^{\prime}(z)\right)+2 \operatorname{Re} E(\zeta) \\
& =-\operatorname{Re} L\left(z F^{\prime}(z) \frac{\zeta+z}{\zeta-z}\right) \leqq 0 .
\end{aligned}
$$

If $A(F(z))$ is analytic in $\varrho<|z|<1$, then the reflection principle shows $\left[z F^{\prime}(z) / F(z)\right]^{2} A(F(z))$ is analytic up to isolated poles on $|z|=1$. For $z=e^{i t}, \mathrm{~d} z / z=i d t$ and from (4.8) the boundary of $U$ must be a trajectory of $A(w) d w^{2} / w^{2}$.

Theorem 4.2. Let $\Psi$ and $F$ be as in the previous theorem. Then $A(w) d w^{2} / w^{2}$ is invariant under the mapping $w \mapsto 1 / w$. Suppose $A(w)$ is analytic up to isolated poles and is not identically zero. If $w_{0}$ and $w_{0}^{-1}$ are not in $D=F(U)$, then at least one is on the boundary of $D$. The points 1 and -1 are on the boundary of $D$. The function $G(z)=F(z)+1 / F(z)$ is univalent in $U$ and maps $U$ onto a domain $D_{1}$ (containing $\infty$ ) whose closure is the entire complex sphere.

Proof. We remark that these observations were made by Nelson [26] for the case of the particular extremal problems he considered.

The first result is immediate from the definition of $A(w)$ in (4.5). The second follows from the fact that if $w_{0}$ and $w_{0}^{-1}$ were exterior to $D$, Theorem 2.3 would apply and

$$
\Psi\left(F^{*}\right)=\Psi(F)+\varepsilon e^{i \alpha} A\left(w_{0}\right)+o(\varepsilon) .
$$

Taking the real part, this implies $A\left(w_{0}\right)=0$. However, this would then have to hold in an entire neighborhood of $w_{0}$, giving a contradiction.

Since 1 and -1 are such that $w_{0}=w_{0}^{-1}$, both points must be on $\partial D$.

Finally $w+1 / w$ has a two-valued inverse, the values being $w$ and $1 / w$. Hence the Bieberbach-Eilenberg property of $D$ makes $w+1 / w$ univalent on $D$. Any point exterior to $D_{1}$ would have to arise from a $w_{0}$ exterior to $D$ such that $w_{0}^{-1}$ was also exterior to $D$. This completes the proof.

4.4. The following theorem is useful in many applications.

The orem 4.3. Let $\Psi$ be a complex valued functional over $\mathscr{E}$ having a continuous Frêchet derivative $L$ as in the hypotheses of Theorem 4.1. Let $T=\{\Psi(F): F \in \mathscr{E}\}$ 
and suppose $F \in \mathscr{E}$ is such that $\Psi(F)$ is on the boundary of $T$. If $E(\zeta)$ is not constant, then there exists a complex $\lambda$ with $|\lambda|=1$ such that $F$ satisfies Theorems 4.1 and 4.2 as if it were locally extremal for $\operatorname{Re} \lambda \Psi(F)$.

Proof. The proof follows the argument found on pages 495, 496 of [30], but we sketch it here since there are some differences in this case. The variation of Theorem 2.2 gives

where

$$
\Psi\left(F^{*}\right)=\Psi(F)+\varepsilon e^{i \alpha} U-\varepsilon e^{-i \alpha} V+o(\varepsilon),
$$

$$
\begin{aligned}
& U=A\left(F\left(z_{0}\right)\right) / F\left(z_{0}\right)-\left(F\left(z_{0}\right) / z_{0} F^{\prime}\left(z_{0}\right)\right)^{2} E\left(z_{0}\right) / F\left(z_{0}\right), \\
& V=\overline{\left(F\left(z_{0}\right) / z_{0} F^{\prime}\left(z_{0}\right)\right)^{2}} \cdot\left[L\left(z F^{\prime}(z)\right)+E\left(1 / \bar{z}_{0}\right)\right] / \overline{F\left(z_{0}\right),}
\end{aligned}
$$

these quantities being computed from (4.5). Now $s=\varepsilon e^{i \alpha} U-\varepsilon e^{-i \alpha} V$ is a linear transformation of the real and imaginary parts of $\varepsilon e^{i \alpha}$ to the real and imaginary parts of $s$. Hence as $\varepsilon$ and $\alpha$ vary, a full neighborhood of $s=0$ will be covered unless the rank of the transformation is less than 2 . Since $\Psi(F)$ is assumed to be a boundary point of $T$, this rank must be less than 2 and $e^{i \alpha} U-e^{-i \alpha} V$ has constant argument for all real $\alpha$. That is, there exists a $\lambda$ with $|\lambda|=1$ so that $\operatorname{Re}\left\{e^{i \alpha} \lambda U-e^{-i \alpha} \lambda V\right\}=$ $=\operatorname{Re} e^{i \alpha}\{\lambda U-\bar{\lambda} \bar{V}\}=0$. Hence $\lambda U=\bar{\lambda} \bar{V}$. Then $F$ satisfies the differential equation

$$
\lambda A(w) \frac{d w^{2}}{w^{2}}=\left[\lambda E(z)+\bar{\lambda} \overline{L\left(z F^{\prime}(z)\right)}+\bar{\lambda} \overline{E(1 / \bar{z})}\right] \frac{d z^{2}}{z^{2}} .
$$

Next, we show that $\lambda L\left(z F^{\prime}(z)\right)$ is real and $B_{\lambda}(\zeta)=\lambda E(\zeta)+\lambda L\left(z F^{\prime}(z)\right)+\overline{\bar{\lambda} E(1 / \bar{\zeta})} \leqq 0$ for $|\zeta|=1$. First, consider a combination of variations (4.1) and (2.6). Let $-1 \leqq u \leqq 1$, $-1 \leqq v \leqq 1$ and set $F_{1}(z)=F\left(e^{i \varepsilon u} z\right)$. Apply variation (2.6) to $F_{1}$ with $\varepsilon$ replaced by $\varepsilon v$. Since $F_{1}=F+O(\varepsilon)$, we find

$$
\begin{aligned}
\Psi\left(F^{*}\right) & =\Psi\left(F_{1}\right)+\varepsilon v\left[e^{i \alpha} U-e^{-i \alpha} V\right]+o(\varepsilon) \\
& =\Psi(F)+i \varepsilon u L\left(z F^{\prime}(z)\right)+\varepsilon v\left[e^{i \alpha} U-e^{-i \alpha} V\right]+o(\varepsilon) \\
& =\Psi(F)+\varepsilon W(u, v)+o(\varepsilon) .
\end{aligned}
$$

Here, $\lambda W(u, v)=u i \lambda L\left(z F^{\prime}(z)\right)+v\left[e^{i \alpha} \lambda U-e^{-i \alpha} \bar{\lambda} \bar{U}\right]+o(\varepsilon)=u i \lambda L\left(z F^{\prime}(z)\right)+2 v i \operatorname{Im}\left\{e^{i \alpha} \lambda U\right\}$. Again, since a neighborhood of 0 cannot be covered, we must have $\lambda L\left(z F^{\prime}(z)\right)$ real. The exception would be if $U \equiv 0$. But then $V \equiv 0$ and hence $E\left(1 / \bar{z}_{0}\right)$ would be constant. Hence $\lambda L\left(z F^{\prime}(z)\right)$ is real and so is $B_{\lambda}(\zeta)$ if $|\zeta|=1$.

In an exactly similar way we may combine the variation (4.2) with (2.6). Then for $\zeta$ with $|\zeta|=1, \Psi\left(F^{*}\right)=\Psi(F)+\varepsilon W_{1}(u, v)+o(\varepsilon)$, where $\varepsilon>0,0 \leqq u \leqq 1,-1 \leqq v \leqq 1$, and $\lambda W_{1}(u, v)=-u \lambda L\left(z F^{\prime}(z)(\zeta+z) /(\zeta-z)\right)+2 v i \operatorname{Im}\left\{e^{i \alpha} \lambda U\right\}$. Again, $\operatorname{Im}\left\{e^{i \alpha} \lambda U\right\}$ is not identically zero. Hence, if the real part of $\lambda L\left(z F^{\prime}(z)(\zeta+z) /(\zeta-z)\right)$ took on different signs for two $\zeta$, then this combination of variations would cover a neighborhood of $\Psi^{\prime}(F)$. Hence $B_{\lambda}(\zeta)=-\operatorname{Re}\left[\lambda L\left(z F^{\prime}(z)(\zeta+z) /(\zeta-z)\right)\right]$ cannot change sign.

When we chose $\lambda$ above, we could have equally well chosen $-\lambda$. Hence by proper choice we can assure that $B_{\lambda}(\zeta) \leqq 0$ for all $\zeta$ with $|\zeta|=1$. Finally, the proof 
of Theorem 4.2 holds with only minor changes. If (4.10) holds, then $\Psi\left(F^{*}\right)$ will again cover a neighborhood of $\Psi(F)$, leading to a contradiction.

4.5. Letting $z \rightarrow 0$ in (4.8) will give a Marty relation for the given extremal problem. This can also be obtained quite simply directly from (2.6).

Theorem 4.4. Let $\Psi$ and $L$ be as in Theorem 4.1. Suppose $F(z)=b_{1} z+b_{2} z^{2}+\ldots$ ... $\in \mathscr{E}$ is locally extremal for $\max \operatorname{Re} \Psi(F)$. Then $F$ satisfies the Marty relation

$$
\left.b_{1} L\left(F ; 1-F(z)^{2}\right)=L\left(F ; F^{\prime}(z)\right)-\overline{L\left(F ; z^{2} F^{\prime}(z)\right.}\right) .
$$

Proof. Put $z_{0}=0$ in (2.6). This relation follows easily.

\section{General extremal problems for pairs}

5.1. The pair property has one useful symmetry. If $\{F, G\}$ is a pair, then so is $\{\overline{F(\bar{z})}, \overline{G(\bar{z})}\}$. Although the pair property is quite restrictive, there is a wide class of transformations available for special normalization. In particular, if $\{F, G\}$ is a pair and $a \neq 0$, then $\left\{a F, a^{-1} G\right\}$ is also a pair. If $g_{1}(z)$ and $g_{2}(z)$ are any univalent functions mapping $U$ into $U$ with $g_{1}(0)=g_{2}(0)=0$, and $\{F, G\}$ is a univalent pair, then $\left\{F\left(g_{1}(z)\right), G\left(g_{2}(z)\right)\right\}$ will be a univalent pair.

Specifically, if $\{F, G\}$ is a univalent pair and $\varrho>0, \alpha_{0}, \alpha_{1}, \alpha_{2}$ are any real numbers, then

$$
\left\{\varrho e^{i \alpha_{0}} F\left(e^{i \alpha_{1}} z\right), \varrho^{-1} e^{-i \alpha_{0}} G\left(e^{i \alpha_{2}} z\right)\right\}
$$

is also a univalent pair. Observe that by proper choice of $\alpha_{0}, \alpha_{1}$, and $\alpha_{2}$, we can use (5.1) to make any coefficient of $F$ and any two coefficients of $G$ real and positive.

We also observe that the rotational and slit variations of (4.1) and (4.2) can be applied independently to each member of a univalent pair.

5.2. The class of univalent pairs is not compact, but it does have some compactness properties. Suppose $\left\{F_{n}, G_{n}\right\}$ is a sequence of univalent pairs. Then by extracting subsequences as necessary we can assume $F_{n}(z) / F_{n}^{\prime}(0) \rightarrow f(z) \in \mathscr{S}$, $G_{n}(z) / G_{n}^{\prime}(0) \rightarrow g(z) \in \mathscr{S}, F_{n}^{\prime}(0) \rightarrow b$, and $G_{n}^{\prime}(0) \rightarrow c$. If $b$ and $c$ are both finite non-zero complex numbers, then the pairs $\left\{F_{n}, G_{n}\right\}$ converge uniformly on compact subsets of $U$ to the univalent pair $\{b f(z), c g(z)\}$.

If $b=\infty$, then quarter theorem and the pair property show that $G_{n}(U) \subset$ $\subset\left\{w:|w|<4 /\left|F_{n}^{\prime}(0)\right|\right\}$ and hence $c=0$. In this case $F_{n}(z) \rightarrow \infty$ (in the sense that it converges to $\infty$ uniformly in compact subsets of $U-\{0\}$ ) and $G_{n}(z) \rightarrow 0$ (uniformly in compact subsets of $U$ ).

If $b=0$, then $F_{n}(z) \rightarrow 0$. However, $G_{n}(z)$ may converge to $\infty, 0$, or some univalent $G(z)$. This is easily seen by considering examples of $F_{n}(z)$ which map $U$ onto $V_{n}=\{w:|w|<1 / n\}$, or onto $V_{n} \cup W\left(1 / n^{2}, n\right)$, or onto $V_{n} \cup W\left(1 / n^{2}, 4\right)$ where $W(\delta, R)=\{w: 0<\operatorname{Re} w<R,|\operatorname{Im} w|<\delta\}$. 
If $b$ is finite and non-zero, then the $F_{n}(z)$ converge to some univalent $F$. The $G_{n}$ may converge to a univalent $G$, in which case $\{F, G\}$ is a pair, or the $G_{n}$ may converge to 0 .

Individual extremal problems must be considered carefully to see whether an extremal univalent pair does indeed exist. For example, $\left(\left|b_{1}\right|+\left|c_{1}\right|\right)$ is easily seen to have no maximum in the set of all pairs.

5.3. Let $\Psi\left(F_{1}, F_{2}\right)$ be a continuous complex valued functional defined over all univalent pairs $\left\{F_{1}, F_{2}\right\}$. We assume that $\Psi$ has a Frêchet derivative at $\left\{F_{1}, F_{2}\right\}$. That is,

$$
\Psi\left(F_{1}+\varepsilon H_{1}, F_{2}+\varepsilon H_{2}\right)=\Psi\left(F_{1}, F_{2}\right)+\varepsilon L_{1}\left(F_{1}, F_{2} ; H_{1}\right)+\varepsilon L_{2}\left(F_{1}, F_{2} ; H_{2}\right)+o(\varepsilon)
$$

where $L_{1}$ and $L_{2}$ are continuous linear functionals of $H_{1}$ and $H_{2}$, respectively. We will usually suppress the dependence on $F_{1}$ and $F_{2}$ and write these simply as $L_{1}\left(H_{1}\right)$ and $L_{2}\left(H_{2}\right)$. We will say that a univalent pair $\left\{F_{1}, F_{2}\right\}$ is (locally) extremal for $\operatorname{Re} \Psi\left(F_{1}, F_{2}\right)$ if $\operatorname{Re} \Psi\left(F_{1}^{*}, F_{2}^{*}\right) \leqq \operatorname{Re} \Psi\left(F_{1}, F_{2}\right)$ for all (nearby) univalent pairs $\left\{F_{1}^{*}, F_{2}^{*}\right\}$. Here "nearby" is in the sense of convergence on compact subsets of $U$.

Suppose that the univalent pair $\left\{F_{1}, F_{2}\right\}$ is locally extremal for $\operatorname{Re} \Psi\left(F_{1}, F_{2}\right)$. From Theorem 3.1 and (5.2) we must therefore have

$\operatorname{Re} e^{i \alpha}\left\{L_{1}\left(\frac{F_{1}}{F_{1}-w_{0}}\right)-L_{2}\left(\frac{F_{2}^{2}}{1-w_{0} F_{2}}\right)-\left(\frac{F_{1}\left(z_{0}\right)}{z_{0} F_{1}^{\prime}\left(z_{0}\right)^{2}}\right)\left[L_{1}\left(\frac{z F_{1}^{\prime}(z)}{z-z_{0}}\right)-\overline{L_{1}\left(\frac{z^{2} F_{1}^{\prime}(z)}{1-\bar{z}_{0} z}\right)}\right]\right\} \leqq 0$

for any $z_{0} \in U$ and any real $\alpha$. Here $w_{0}=F_{1}\left(z_{0}\right)$ and we conjugated the term containing $e^{-i \alpha}$ in (3.10).

Define

$$
\begin{cases}D_{1}(w)=L_{1}\left(\frac{w F_{1}}{F_{1}-w}\right) & D_{2}(w)=L_{2}\left(\frac{w F_{2}}{F_{2}-w}\right) \\ E_{1}(\zeta)=L_{1}\left(\frac{\zeta z F_{1}^{\prime}(z)}{z-\zeta}\right) & E_{2}(\zeta)=L_{2}\left(\frac{\zeta z F_{2}^{\prime}(z)}{z-\zeta}\right) \\ A_{1}(w)=D_{1}(w)+L_{2}\left(F_{2}\right)+D_{2}(1 / w) & A_{2}(w)=D_{2}(w)+L_{1}\left(F_{1}\right)+D_{1}(1 / w) \\ \left.B_{1}(\zeta)=E_{1}(\zeta)+\overline{L_{1}\left(z F_{1}^{\prime}(z)\right.}\right)+\overline{E_{1}(1 / \zeta)} & \left.B_{2}(\zeta)=E_{2}(\zeta)+\overline{L_{2}\left(z F_{2}^{\prime}(z)\right.}\right)+\overline{E_{2}(1 / \bar{\zeta})} .\end{cases}
$$

Proceeding almost exactly as in the proof Theorems 4.1 and 4.2, noting that the roles of $F_{1}$ and $F_{2}$ can be interchanged in Theorem 3.1, one easily verifies:

The orem 5.1. Let $\Psi\left(F_{1}, F_{2}\right)$ be a continuous complex valued functional defined over all univalent pairs, having a continuous linear Frêchet derivative defined as in (5.2). Let $A_{1}, A_{2}, B_{1}$, and $B_{2}$ be defined as in (5.4). Suppose the univalent pair $\left\{F_{1}, F_{2}\right\}$ is locally extremal for $\operatorname{Re} \Psi\left(F_{1}, F_{2}\right)$. Then $L_{1}\left(z F_{1}^{\prime}(z)\right)$ and $L_{2}\left(z F_{2}^{\prime}(z)\right)$ are real; $B_{1}(z)$ 
and $B_{2}(z)$ are real and non-positive for $|z|=1$; and $F_{1}$ and $F_{2}$ satisfy the differential equations

$$
\left\{\begin{array}{l}
\left(\frac{z F_{1}^{\prime}(z)}{F_{1}(z)}\right)^{2} A_{1}\left(F_{1}(z)\right)=B_{1}(z), \quad z \in U \\
\left(\frac{z F_{2}^{\prime}(z)}{F_{2}(z)}\right)^{2} A_{2}\left(F_{2}(z)\right)=B_{2}(z), \quad z \in U .
\end{array}\right.
$$

If either $A_{1}(w)$ or $A_{2}(w)$ is analytic up to isolated singularities and not identically zero, then there is no $w_{0}$ exterior to $F_{1}(U)$ with $w_{0}^{-1}$ simultaneously exterior to $F_{2}(U)$.

We remark that the last conclusion of theorem is equivalent to saying that the closure of $\left[F_{1}(U)\right] \cup\left[F_{2}(U)\right]^{\prime}$ is the entire complex sphere, where we denote $B^{\prime}=\{w: 1 / w \in B\}$.

5.4. In most extremal problems, $A_{1}, A_{2}, B_{1}$, and $B_{2}$ are analytic up to isolated poles (and not identically zero). Since $d z^{2} / z^{2}<0$ on $|z|=1$, it follows that the boundaries of $F_{1}(U)$ and $F_{2}(U)$ lie on the trajectories

$$
A_{1}(w) \frac{d w^{2}}{w^{2}} \geqq 0, \quad A_{2}(w) \frac{d w^{2}}{w^{2}} \geqq 0,
$$

respectively. These boundaries must consist of a simple closed curve plus some possible slits and must be made up of analytic curves and arcs. Any branching can occur only at the critical points of $A_{1}$ and $A_{2}$. Since $w \mapsto 1 / w$ carries the outer boundary of $F_{1}(U)$ to the outer boundary of $F_{2}(U)$, we must have $A_{1}(w) / A_{2}(1 / w)$ real and positive for $w$ on the analytic arcs making up the outer boundary of $F_{1}(U)$. In practice, we find for most simple problems that after suitable normalization $A_{1}(w)=A_{2}(1 / w)$.

5.5. The obvious analog of Theorem 4.3 will hold for pairs. The proof is essentially the same.

Similarly, Marty relations will hold for an extremal pair. Putting $z_{0}=0$ in Theorem 3.1 gives

$$
\begin{aligned}
& F_{1}^{\prime}(0)\left[L_{1}(1)-L_{2}\left(F_{2}^{2}\right)\right]=L_{1}\left(F_{1}^{\prime}(z)\right)-\overline{L_{1}\left(z^{2} F_{1}^{\prime}(z)\right)}, \\
& F_{2}^{\prime}(0)\left[L_{2}(1)-L_{1}\left(F_{1}^{2}\right)\right]=L_{2}\left(F_{2}^{\prime}(z)\right)-\overline{L_{2}\left(z^{2} F_{2}^{\prime}(z)\right)} .
\end{aligned}
$$

Again these Marty relations are interesting but do not seem to be generally useful.

5.6. It is useful to demonstrate the use of Theorem 5.1 in a very simple case. Let $F_{1}(z)=b_{1} z+b_{2} z^{2}+\ldots, F_{2}(z)=c_{1} z+c_{2} z^{2}+\ldots$ and consider the problem of maximizing $\left|b_{1} c_{1}\right|$ among all univalent pairs. Here the solution $\left|b_{1} c_{1}\right| \leqq 1$ is well known [1] and is sharp, for example for the pair $F_{1}(z)=F_{2}(z)=z$. However, let us analyze the problem using the methods of this section.

From (5.1) we may assume $b_{1}>0$ and $c_{1}>0$, and hence set $\Psi\left(F_{1}, F_{2}\right)=$ $=\chi_{1}\left(F_{1}\right) \chi_{2}\left(F_{2}\right)$, where $\chi_{v}(H(z))$ represents the coefficient of $z^{v}$ in the series expansion 
of $H(z)$. Let $\alpha=\sup \operatorname{Re}\left\{\chi_{1}\left(F_{1}\right) \chi_{1}\left(F_{2}\right):\left\{F_{1}, F_{2}\right\}\right.$ is a univalent pair $\}$. There exists a sequence of pairs $\left\{F_{1, n}, F_{2, n}\right\}$ with $\chi_{1}\left(F_{1, n}\right)=1, \chi_{1}\left(F_{2, n}\right) \rightarrow \alpha$. The quarter theorem shows that the $F_{2, n}$ are bounded, hence $\alpha$ is finite. Since $\alpha>0$, the $F_{2, n}$ do not converge to 0 . Hence an extremal pair exists for this $\Psi$. Observe that the important fact here is that $\Psi$ is invariant under $\left\{F_{1}, F_{2}\right\} \mapsto\left\{a F_{1}, a^{-1} F_{2}\right\}$.

For $\Psi\left(F_{1}, F_{2}\right)=\chi_{1}\left(F_{1}\right) \chi_{1}\left(F_{2}\right)$, one easily verifies that $L_{1}\left(H_{1}\right)=c_{1} \chi_{1}\left(H_{1}\right)$, $L_{2}\left(H_{2}\right)=b_{1} \chi_{1}\left(H_{2}\right)$ where $F_{1}(z)=b_{1} z+\ldots$ and $F_{2}(z)=c_{1} z+\ldots$. Then from (5.4) $A_{1}(w)=A_{2}(w)=B_{1}(z)=B_{2}(z)=-b_{1} c_{1}$ and hence the extremal pair satisfy

$$
\left(\frac{z F_{1}^{\prime}(z)}{F_{1}(z)}\right)^{2}=1 \quad\left(\frac{z F_{2}^{\prime}(z)}{F_{2}(z)}\right)^{2}=1 .
$$

Thus $F_{1}(z)=b_{1} z, F_{2}(z)=c_{1} z$, and the relationship of the boundaries of $F_{1}(U)$ and $F_{2}(U)$ requires $\left|c_{1}\right|=1 /\left|b_{1}\right|$, i.e., $\alpha=\left|b_{1} c_{1}\right|=b_{1} c_{1}=1$ for the extremal pair.

\section{The value sets for $F \in \mathscr{E}$}

6.1. In 1954 Jenkins showed [18] that if $F \in \mathscr{E}$ and $\left|z_{0}\right|=r$, then $\left|F\left(z_{0}\right)\right| \leqq$ $\leqq r /\left(1-r^{2}\right)^{1 / 2}$, with the maximum being achieved at $z_{0}=i e^{-i \varphi} r$ by the function

$$
F_{r, \varphi}(z)=\frac{\left(1-r^{2}\right)^{1 / 2} e^{i \varphi} z}{1+i r e^{i \varphi} z} .
$$

This function maps $U$ onto the interior of a circle centered on the imaginary axis and passing through the points \pm 1 . Such a circle is invariant under the mapping $w \mapsto 1 / w$ and hence $F_{r, \varphi} \in \mathscr{E}$.

In this section we study the set of possible values of a Bieberbach-Eilenberg functions at a fixed point in $U$. Define, for any $r$ with $0<r<1$

$$
V(r)=\{F(r): F \in \mathscr{E}\} .
$$

If $F(z) \in \mathscr{E}$, then so is $F\left(\varrho e^{i \alpha} z\right)$ for $0<\varrho \leqq 1$ and any real $\alpha$. Hence $V(r)=$ $=\left\{F\left(r e^{i \alpha}\right): F \in \mathscr{E}\right\}=\{F(z): F \in \mathscr{E}$ and $0<|z| \leqq r\}$. If $F \in \mathscr{E}$, then so are $-F(z)$ and $\overline{F(\bar{z})}$. Hence $V(r)$ is symmetric with respect to reflection in the real and imaginary axes. It thus suffices to study $V(r)$ in the first quadrant. The points $0,+1,-1$ are never in any $V(r)$, but each $V(r), 0<r<1$, contains a punctured neighborhood of 0. Each $V(r)$ is bounded (from the Jenkins result or using the fact that $F(z) / F^{\prime}(0)$ is in $\mathscr{S}$ and $\left|F^{\prime}(0)\right| \leqq 1$ so $\left.|F(r)| \leqq r /\left(1-r^{2}\right)\right)$. Thus $V(r) \cup\{0\}$ is compact. It is easy to construct examples of Bieberbach-Eilenberg domains with boundaries made up of circular arcs containing any preassigned $b \neq 0,+1,-1$. Thus, each such $b$ is in some $V(r)$. If $F(r)=b$, then $F$ maps a neighborhood of $r$ onto a neighborhood of $b$. Hence $b$ is an interior point of $V\left(r_{1}\right)$ for any $r_{1}>r$. That is, the domains $V(r) \cup\{0\}$ are strictly monotone increasing and $r_{0}=\inf \{r: b \in V(r)\}$ is such that $b \in \partial V\left(r_{0}\right)$. 
6.2. Let $b \neq 0,1$ be a boundary point of $V(r)$ in the first quadrant (i.e., $0 \leqq \arg b \leqq$ $\leqq \pi / 2$ ). Then $\log b$ lies on the boundary of the set of $\log F(r)$ and from Theorem 4.3, if $F \in \mathscr{E}$ with $F(r)=b$, then $F$ will be locally extremal for $\operatorname{Re} \lambda \log F(r)$ for some $\lambda$ with $|\lambda|=1$. (It is not essential to take the logarithm here, but it offers a minor convenience to do so.) Thus we consider $\Psi(F)=\lambda \log F(r)$. Then $L(H)=\lambda H(r) / F(r)$. If $F$ is locally extremal for $\operatorname{Re} \lambda \log F(r)$, then from Theorem 4.1, $F$ satisfies

$$
\frac{\lambda\left(1-b^{2}\right) d w^{2}}{w(b-w)(1-b w)}=\frac{\lambda c\left(1-r^{2}\right) d z^{2}}{z(r-z)(1-r z)}, \quad w=F(z), \quad z \in U,
$$

where $b=F(r)$ and $c=r F^{\prime}(r) / F(r)$. Theorem 4.1 shows $\lambda c$ is real, and since $B(1)=-\lambda c\left(1-r^{2}\right) /(1-r)^{2}, \lambda c>0$. Observe that $\lambda=1$ corresponds to maximizing $|F(r)|$ for $F \in \mathscr{E}$.

\subsection{Set}

$$
\Omega=\Omega(w)=\int_{0}^{w}[w(b-w)(1-b w)]^{-1 / 2} d w .
$$

This an elliptic integral and hence the inverse of a doubly periodic function. The critical points are $0, b, b^{-1}$, and $\infty$. One period, $2 \hat{\omega}_{1}$, will be twice the integral from 0 to $b$ along the line joining these points. Setting $w=b t^{2}$, we find

$$
\begin{aligned}
\hat{\omega}_{1} & =2 \int_{0}^{1}\left[\left(1-t^{2}\right)\left(1-b^{2} t^{2}\right)\right]^{-1 / 2} d t \\
& =2 K(b)
\end{aligned}
$$

where $K(b)$ is the normal complete elliptic integral of the first kind (in Jacobi's form). This is uniquely defined for any $b \neq 0,1$ in the first quadrant if we specify that its real part is positive to fix the sign of the root and define it by continuity for $b$ real and greater than 1 . We remark that in the last case there is no canonical path of integration. Passing above or below $b^{-1}$ results in complex conjugate determinations of $K(b)$. We choose the determination resulting from passing above.

A second period of (6.4) is $2 \hat{\omega}_{2}$, equal to twice the integral along the circular arc from $b$ to $b^{-1}$ through 1 . This integral is twice the integral from $b$ to 1 along this path since $d \Omega$ is invariant under the mapping $w \mapsto 1 / w$. Set $b_{1}=(1-b) /(1+b)$ and $w=\left(1-b_{1} t\right) /\left(1+b_{1} t\right)$. Then

$$
\begin{aligned}
\hat{\omega}_{2} & =\frac{4 i}{(1+b)} \int_{0}^{1}\left[\left(1-t^{2}\right)\left(1-b_{1}^{2} t^{2}\right)\right]^{-1 / 2} d t \\
& =\frac{4 i}{1+b} K\left(b_{1}\right) .
\end{aligned}
$$

Landen's (or Gauss') transformation gives $2 K((1-b) /(1+b))=(1+b) K\left(\sqrt{1-b^{2}}\right)=$ 
$=(1+b) K^{\prime}(b)$ where $K^{\prime}(b)$ is the associated complete elliptic integral. See [8] for example. Thus

$$
\hat{\omega}_{2}=2 i K^{\prime}(b) .
$$

Other pairs of periods of (6.4) will be related to $\hat{\omega}_{1}$ and $\hat{\omega}_{2}$ by unimodular transformations and will correspond to integration over homotopically different paths in the plane less the critical points $0, b, b^{-1}$.

The line segment joining 0 to $\hat{\omega}_{1}$ in the $\Omega$-plane corresponds to a path $\hat{\gamma}_{1}$ joining 0 to $b$ in the $w$-plane, along which $d \Omega$ has a constant argument, and which is homotopic to the line segment joining 0 to $b$. (All homotopies are relative to $\mathbf{C}-\left\{0, b, b^{-1}\right\}$.) Similarly the line joining $\hat{\omega}_{1}$ to $\hat{\omega}_{1}+(1 / 2) \hat{\omega}_{2}$ in the $\Omega$-plane corresponds to a path $\hat{\gamma}_{2}$ joining $b$ to 1 in the $w$-plane, along which $d \Omega$ has a constant argument, and which is homotopic to the line segment joining $b$ to 1 . Let $\hat{\gamma}_{2}^{\prime}$ and $\hat{\gamma}_{1}^{\prime}$ be the images of $\hat{\gamma}_{2}$ and $\hat{\gamma}_{1}$ under the mapping $w \mapsto 1 / w$. Then $\hat{\gamma}_{1}+\hat{\gamma}_{2}+\hat{\gamma}_{2}^{\prime}+\hat{\gamma}_{1}^{\prime}$ is a path from 0 to $\infty$ through $b, 1$, and $b^{-1}$ along each separate arc of which $d \Omega$ is constant. (6.4) defines a univalent mapping of the complement of this path onto the parallelogram with vertices $\pm \hat{\omega}_{1}, \pm \hat{\omega}_{1}+\hat{\omega}_{2}$, in which $b$ corresponds to $\pm \hat{\omega}_{1}, 1$ to $\pm \hat{\omega}_{1}+(1 / 2) \hat{\omega}_{2}$, $b^{-1}$ to $\pm \omega_{1}+\omega_{2}$, and $\infty$ to $\hat{\omega}_{2}$.

This parallelogram is half of the entire period parallelogram. Starting on the "other side" of $\hat{\gamma}_{1}$, we see that (6.4) also maps the same split plane univalently onto the other half of the period parallelogram. Finally, observe that the mapping $w \mapsto 1 / w$ corresponds to symmetry with respect to $\hat{\omega}_{1}+(1 / 2) \hat{\omega}_{2}$ or $\hat{\omega}_{1}+(3 / 2) \hat{\omega}_{2}$, modulo the two periods.

6.4. In a similar way we can consider the mapping

$$
Z=\int_{0}^{z}[z(r-z)(1-r z)]^{-1 / 2} d z .
$$

Here $0<r<1$ and this is a much simpler integral. Much as in $\S 6.3$ we find that (6.7) maps the interior of the unit circle slit from 0 to 1 along the real axis univalently onto the interior of the rectangle with vertices $\pm 2 K(r)$ and $\pm 2 K(r)+i K^{\prime}(r)$. This is one fourth of the entire period parallelogram. Here $z=r$ corresponds to $\pm 2 K(r)$ and $z=1$ corresponds to $\pm 2 K(r)+i K^{\prime}(r) . z=-1$ corresponds to $i K^{\prime}(r)$.

6.5. Suppose $w=F(z)$ is analytic and univalent in $U$ and satisfies (6.3) for some $r, \lambda$, and $c$ with $\lambda c>0, F(r)=b$, and with 1 on the boundary of $F(U)$. Since $b$ is interior to $F(U)$, the boundary of $F(U)$ is a trajectory of the left-hand side of (6.3) and hence a simple closed analytic curve which we denote by $\gamma_{3}$. We know 1 lies on $\gamma_{3}$ and since (6.3) is invariant under $w \mapsto 1 / w$, the same is true of $\gamma_{3}$. Therefore $F \in \mathscr{E}$.

Let $\gamma_{1}$ be the image of the segment $[0, r]$ under the mapping $w=F(z)$ and let $\gamma_{2}$ be the image of $[r, 1]$. Then $\gamma_{1}$ is a trajectory of the left-hand side of (6.3) joining 0 to $b$ while $\gamma_{2}$ is an orthogonal trajectory joining $b$ to some point $w_{0} \in \gamma_{3}$. 
The mapping (6.4) carries $\gamma_{1}$ to a line segment $l_{1}$ joining 0 to some point $\omega_{1}$ which will be the same as $\hat{\omega}_{1}$ modulo the period parallelogram. That is, $\omega_{1}=n_{1} \hat{\omega}_{1}+$ $+n_{2} \hat{\omega}_{2}$ where $n_{1}$ is the same odd integer and $n_{2}$ is some even integer. $\gamma_{2}$ maps to a line segment $l_{2}$, orthogonal to $l_{1}$, joining $\omega_{1}$ to some point $\omega_{1}+\omega_{0} . \gamma_{3}$ maps to a line segment $l_{3}$, parallel to $l_{1}$, from $\omega_{1}+\omega_{0}$ to $-\omega_{1}+\omega_{0}$. Thus (6.3) maps the interior of $\gamma_{3}$ less the slit $\gamma_{1}+\gamma_{2}$ to the interior of the rectangle $R$ with vertices $\pm \omega_{1}, \pm \omega_{1}+\omega_{0}$. The line segment $l_{3}$ must contain a point $\omega_{0}^{\prime}$ which is the image of 1 under (6.4). That is, $\omega_{0}^{\prime}=\hat{\omega}_{1} \pm(1 / 2) \hat{\omega}_{2}+2 k \hat{\omega}_{1}+2 j \hat{\omega}_{2}$. It follows that there exists an $\omega_{2}$ such that $\omega_{0}^{\prime}=\omega_{1}+(1 / 2) \omega_{2}$ and $\omega_{2}=n_{3} \hat{\omega}_{1}+n_{4} \hat{\omega}_{2}$ where $n_{3}$ is some even integer and $n_{4}$ is some odd integer. The pair $2 \omega_{1}, 2 \omega_{2}$ is a pair of periods for the inverse of (6.4).

The above mapping can be reflected in $l_{3}$. The exterior of $\gamma_{3}$ slit from $w_{0}^{-1}$ to $\infty$ along the image of $\gamma_{1}+\gamma_{2}$ under $w \mapsto 1 / w$ will map to a rectangle congruent to $R$. and having a subsegment of $l_{3}$ in common on the boundary. It follows that the area of $R$ is one quarter of the area of the primitive period parallelogram and hence $2 \omega_{1}, 2 \omega_{2}$ is a primitive pair of periods. That is

$$
\begin{cases}\omega_{1}=n_{1} \hat{\omega}_{1}+n_{2} \hat{\omega}_{2} & n_{1}, n_{4} \text { odd integers } \\ \omega_{2}=n_{3} \hat{\omega}_{1}+n_{4} \hat{\omega}_{2} & n_{2}, n_{3} \text { even integers } \\ & n_{1} n_{4}-n_{2} n_{3}=1 .\end{cases}
$$

Here $n_{1} n_{4}-n_{2} n_{3} \neq-1$ since the mappings involved are all conformal. The transformation (6.8) thus belongs to the congruence subgroup modulo 2 of the full set of unimodular transformations.

Since $w=F(z)$ satisfies (6.3), we must have $\left[\lambda\left(1-b^{2}\right)\right]^{1 / 2} \Omega=\left[\lambda c\left(1-r^{2}\right)\right]^{1 / 2} Z$. Set $B=\left[\lambda\left(1-b^{2}\right)\right]^{1 / 2}, C=\left[\lambda c\left(1-r^{2}\right)\right]^{1 / 2}$. Then $B \omega_{1}=2 C K(r)$ and $B\left(\omega_{1}+\omega_{0}\right)=$ $=C\left(2 K(r)+i K^{\prime}(r)\right)$. Combining these, $B \omega_{0}=i C K^{\prime}(r)$. Since $\lambda c>0, C>0$ (choosing the proper root). Multiplication of $\Omega$ by $B$ therefore rotates the rectangle $R$ so that the image of $l_{1}$ lies on the real axis, and the image of $l_{3}$ is parallel to the real axis. Hence $\operatorname{Im}\left\{B \omega_{0}\right\}=\operatorname{Im}\left\{B \omega_{0}^{\prime}\right\}=\operatorname{Im}\left\{B \cdot(1 / 2) \omega_{2}\right\}=\operatorname{Im}\left\{C i K^{\prime}(r)\right\}=C K^{\prime}(r)$. Hence we have shown that the following two relations hold:

$$
\begin{gathered}
{\left[\lambda\left(1-b^{2}\right)\right]^{1 / 2} \omega_{1}=\left[\lambda c\left(1-r^{2}\right)\right]^{1 / 2} 2 K(r)>0,} \\
\operatorname{Im}\left\{\frac{\omega_{2}}{\omega_{1}}\right\}=\frac{K^{\prime}(r)}{K(r)} .
\end{gathered}
$$

6.6. We now state the main theorem.

Theorem 6.1. Let $b \neq 0,1$ with $0 \leqq \arg b \leqq \pi / 2$ be given. Let $K(b)$ and $K^{\prime}(b)$ be the normal and associated complete elliptic integrals of the first kind, with sign determinations made so that $\operatorname{Re} K(b) \geqq 0$ and $\operatorname{Re}\left\{K^{\prime}(b) / K(b)\right\}>0$, and with $K(b)$ defined by continuity for $b$ real and greater than 1 . Then there exists a unique $r_{b}$ with 
$0<r_{b}<1$, a unique $\lambda_{b}$ with $\left|\lambda_{b}\right|=1$, a unique $c_{b} \neq 0$, and a function $F_{b}(z) \in \mathscr{E}$ such that $F_{b}\left(r_{b}\right)=b$ and

$$
\begin{gathered}
\operatorname{Re}\left\{\frac{K^{\prime}(b)}{K(b)}\right\}=\frac{K^{\prime}\left(r_{b}\right)}{K\left(r_{b}\right)}, \\
{\left[\lambda_{b}\left(1-b^{2}\right)\right]^{1 / 2} K(b)>0,} \\
\lambda_{b} c_{b}>0, \\
{\left[\lambda_{b}\left(1-b^{2}\right)\right]^{1 / 2} K(b)=\left[\lambda_{b} c_{b}\left(1-r^{2}\right)\right]^{1 / 2} K\left(r_{b}\right) .}
\end{gathered}
$$

Furthermore, $w=F_{b}(z)$ satisfies the differential equation (6.3) with $\lambda=\lambda_{b}, r=r_{b}$, $c=c_{b}=r_{b} F_{b}^{\prime}\left(r_{b}\right) / F_{b}\left(r_{b}\right)$. The given $b$ is on the boundary of $V\left(r_{b}\right)$ and $F_{b}$ is the unique function in $\mathscr{E}$ with $F\left(r_{b}\right)=b$ unless $b$ is real and greater than 1 , in which case there is exactly one more, the function $\overline{F_{b}(\bar{z})}$.

Proof. Since $K^{\prime}(r) / K(r)$ is strictly monotone decreasing from $\infty$ to 0 as $r$ increases from 0 to 1 , there is a unique $r_{b}, 0<r_{b}<1$, satisfying (6.10). Then there is a unique $\lambda_{b}$ with $\left|\lambda_{b}\right|=1$ satisfying (6.11), and a unique $c_{b}$ satisfying (6.12) and (6.13).

Let $\hat{\gamma}_{3}$ be the unique trajectory of the left-hand side of (6.3), with $\lambda=\lambda_{b}$, through $w=1$. This is a simple closed analytic curve, invariant under the mapping $w \mapsto 1 / w$, whose image by (6.4) is the line segment joining $\pm \hat{\omega}_{1}+\hat{\omega}_{2} / 2$. The values which have been chosen are such that (6.4), (6.7), and $\left[\lambda_{b}\left(1-b^{2}\right)\right]^{1 / 2} \Omega=\left[\lambda_{b} c_{b}\left(1-r_{b}^{2}\right)\right]^{1 / 2} Z$ together define a univalent mapping $w=F_{b}(z)$ of $U$ slit from 0 to 1 along the real axis to the interior of $\hat{\gamma}_{3}$ slit by $\hat{\gamma}_{1}+\hat{\gamma}_{2}^{\prime}$ where $\hat{\gamma}_{2}^{\prime}$ is an orthogonal trajectory of the lefthand side of (6.3) joining $b$ to some point of $\hat{\gamma}_{3}$. This function is easily seen to be analytic at each point of the segment $[0,1)$ and hence can be continued to be univalent in all of $U$. Since $\hat{\gamma}_{3}$ is invariant under $w \mapsto 1 / w, F_{b} \in \mathscr{E}$. The method of construction insures that $F_{b}\left(r_{b}\right)=b$. Letting $z \rightarrow r$ in (6.3) gives $c_{b}=r_{b} F_{b}^{\prime}\left(r_{b}\right) / F_{b}\left(r_{b}\right)$.

Next we show that $b \in \partial V\left(r_{b}\right)$. We know $b \in \partial V(r)$ for some $r$ and since $F_{b}\left(r_{b}\right)=b$,

$$
r_{b} \geqq r \text {. }
$$

If $b \in \partial V(r)$, then there exists an $F \in \mathscr{E}$ with $F(r)=b$ satisfying (6.3) and with the boundary of $F(U)$ passing through 1 . From the discussion of $\S 6.5$, there must exist a pair of primitive periods $2 \omega_{1}, 2 \omega_{2}$, such that (6.8) and (6.9) hold. Set $\tau=\omega_{2} / \omega_{1}, \tau_{b}=\hat{\omega}_{2} / \hat{\omega}_{1}=i K^{\prime}(b) / K(b)$. Then

$$
\tau=\frac{n_{4} \tau_{b}+n_{3}}{n_{2} \tau_{b}+n_{1}}
$$

with $n_{1}, n_{2}, n_{3}$, and $n_{4}$ as in (6.8).

Now $\tau_{b}=i K^{\prime}(b) / K(b)$ lies in the region $\operatorname{Im} \tau>0,0 \leqq \operatorname{Re} \tau \leqq 1,|\tau-1 / 2| \geqq 1 / 2$, as is shown, for example, in [10]. It is known (see [15] for example) that this is part of the 
fundamental domain of the congruence subgroup, but we can verify this easily and obtain information on uniqueness at the same time.

From (6.15), $\operatorname{Im} \tau=\operatorname{Im} \tau_{b} /\left|n_{2} \tau_{b}+n_{1}\right|^{2}$. Set $\tau_{b}=x+i y$. Then $\left|\tau_{b}-1 / 2\right| \geqq 1 / 2$ is equivalent to $x^{2}-x+y^{2} \geqq 0$. But then $\left|n_{2} \tau_{b}+n_{1}\right|^{2}=n_{2}^{2}\left(x^{2}-x+y^{2}\right)+\left(n_{1}+n_{2}\right)^{2} x+$ $+n_{1}^{2}(1-x) \geqq x+(1-x)=1$ since $n_{1}$, being odd, is non-zero and $n_{1}+n_{2}$ is similarly non-zero. Therefore, $\operatorname{Im} \tau \leqq \operatorname{Im} \tau_{b}$ with strict inequality holding except when $n_{1}= \pm 1$, $n_{2}=0$, or when $n_{1}= \pm 1, n_{2}=\mp 2,\left|\tau_{b}-1 / 2\right|=1 / 2$.

In the first case, $\omega_{1}= \pm \hat{\omega}_{1}$ and from (6.9), $\lambda=\lambda_{b}$. It follows that $F(U)$ is bounded by $\hat{\gamma}_{3}$, the unique trajectory of the left-hand side of (6.3) through $w=1$, and that $\gamma_{1}=\hat{\gamma}_{1}$. Hence $F(z)=F_{b}(z)$.

In the second case, $b \in(1, \infty), \omega_{1}= \pm \hat{\omega}_{1} \mp 2 \hat{\omega}_{2}$. The two sign choices give the same $\gamma_{1}$ and $\gamma_{3}$, hence only one $F(z)$. It is easily seen that this transformation corresponds to taking the path of integration in computing $K(b)$ "below" $1 / b$, and $F(z)=\overline{F_{b}(\bar{z})}$ is the unique function giving the mapping.

In every other case $K^{\prime}(r) / K(r)=\operatorname{Im} \tau<\operatorname{Im} \tau_{b}=K^{\prime}\left(r_{b}\right) / K\left(r_{b}\right)$. This implies $r>r_{b}$ which contradicts (6.14). This completes the proof of the theorem.

6.7. If $b$ is given, (6.10) determines the $r$ so that $b \in \partial V(r)$. Similarly, if $r$ is given, (6.10) determines the set of $b$ which lie on $\partial V(r)$. In both cases tables such as [10] are useful. It is only if $\lambda$ and $r$ are given, that there is some difficulty. The conditions of Theorem 6.1 in theory will determine $b$ but in practice this may be impossible. However, in a few special cases something can be done.

If $\lambda=1$, corresponding to the problem of maximizing $|F(r)|$, then (6.11) requires

$$
\int_{0}^{1}\left[\frac{1-b^{2}}{1-b^{2} t^{2}}\right]^{1 / 2} \frac{d t}{\left[1-t^{2}\right]^{1 / 2}}>0
$$

Here we are computing the weighted average of a set of complex numbers along, a curve. The values of $\left(1-b^{2}\right) /\left(1-b^{2} t^{2}\right)$ lie on the circular arc from $\left(1-b^{2}\right)$ to 1 which, if continued, would pass through 0 . Thus if $b^{2}$ is not real, then the values of $\left[\left(1-b^{2}\right) /\left(1-b^{2} t^{2}\right)\right]^{1 / 2}$ lie entirely in one half plane and (6.16) can hold only if $b^{2}$ is real. That is, only if $b$ is positive or pure imaginary. Further, $b$ positive and greater than 1 would also make (6.16) impossible.

Suppose $b$ is real with $0<b<1$. Then (6.10) requires $r_{b}=b,(6.11)$ shows $\lambda_{b}>0$, and the unique $F_{b}(z)=z$.

Suppose $b=i \beta, \beta>0$. Then using the imaginary modulus transformation and the reciprocal modulus transformation (see [8], for example), $K(b)=K(i \beta)=\beta_{1}^{\prime} K\left(\beta_{1}\right)$ and $K^{\prime}(b)=K\left(\sqrt{1+\beta^{2}}\right)=K\left(1 / \beta_{1}^{\prime}\right)=\beta_{1}^{\prime}\left[K\left(\beta_{1}^{\prime}\right)+i K^{\prime}\left(\beta_{1}^{\prime}\right)\right]=\beta_{1}^{\prime}\left[K^{\prime}\left(\beta_{1}\right)+i K\left(\beta_{1}\right)\right]$ where $\beta_{1}=\beta / \sqrt{1+\beta^{2}}$ and $\beta_{1}^{\prime}=1 / \sqrt{1+\beta^{2}}$. Hence $(6.10)$ requires $K^{\prime}(r) / K(r)=K^{\prime}\left(\beta_{1}\right) / K\left(\beta_{1}\right)$ or $r=r_{b}=\beta_{1}$. This is equivalent to $\beta=r / \sqrt{1-r^{2}}$. Since $\beta>r$ in this case, this gives the maximum for $F(r)$ and we have Jenkin's result. 
6.8. It is interesting to look at what can be said about $r_{b}$ for $b$ real and greater than 1 . Here the transformations give $K(b)=(1 / b)\left[K(1 / b)+i K^{\prime}(1 / b)\right]$ and $K^{\prime}(b)=$ $=K\left(i \sqrt{b^{2}-1}\right)=(1 / b) K\left(\sqrt{b^{2}-1} / b\right)=(1 / b) K^{\prime}(1 / b)$. Hence $(6.10)$ requires $r=r_{b}$ where

$$
\frac{K^{\prime}(r)}{K(r)}=\frac{\sigma}{1+\sigma^{2}}, \quad \sigma=\frac{K^{\prime}(1 / b)}{K(1 / b)} .
$$

As $b$ increases from 1 to $\infty, \sigma$ increases from 0 to $\infty$, and $\sigma /\left(1+\sigma^{2}\right)$ increases from 0 to a maximum of $1 / 2$ when $\sigma=1$ and then decreases to 0 again. If $r_{0}$ is such that $K^{\prime}\left(r_{0}\right) / K\left(r_{0}\right)=1 / 2$, then for $r<r_{0}, V(r)$ does not intersect $(1, \infty)$. For $r=r_{0}, V(r)$ will have the single point $b=\sqrt{2}$ in common with $(1, \infty)$, and for $r>r_{0}, \partial V(r)$ will intersect $(1, \infty)$ in exactly two points. Since $K^{\prime}(k)=2 K(k)$ when $k=3-2 \sqrt{2}$, $r_{0}=2(3 \sqrt{2}-4)^{1 / 2}=0.98517 \ldots$.

Finally, if $b=e^{i \theta}$, then $K(b)=(1 / 2) e^{-i \theta / 2}\left[K(\cos (\theta / 2))+i K^{\prime}(\cos (\theta / 2))\right]$ and $K^{\prime}(b)=e^{-i \theta / 2} K^{\prime}(\cos (\theta / 2))$. Hence $r_{b}=r$ where

$$
\frac{K^{\prime}(r)}{K(r)}=\frac{2 \varrho}{1+\varrho^{2}}, \quad \varrho=\frac{K^{\prime}(\cos \theta / 2)}{K(\cos \theta / 2)} .
$$

From this one easily verifies that $V(r)$ is contained inside the unit disc if $r<r_{1}=\sqrt{2} / 2$. If $r=r_{1}, V(r)$ is tangent to the unit circle at $\pm i$. If $r_{1}<r<1, \partial V(r)$ intersects the unit circle at precisely one point in each quadrant.

6.9. Figure 1 shows the boundaries of $V(r)$ for the two critical $r$ mentioned above. Only the portion in the first quadrant is shown. The full set is symmetric with respect to reflection in both axes.

The inner curve defines $V\left(r_{1}\right), r_{1}=\sqrt{2} / 2$. The boundary appears nearly vertical near the real axis, but its real part reaches a maximum near $b=0.7077+i 0.13$.

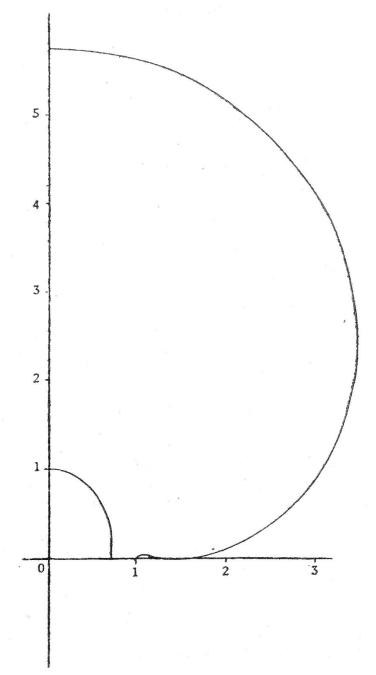

Figure 1 
The outer curve defines $V\left(r_{0}\right), r_{0}=2(3 \sqrt{2}-4)^{1 / 2}$. This boundary is tangent to the real axis at $b=\sqrt{2}$. The very small loop that the boundary makes around the poiut 1 could not be determined very accurately because of the coarseness of the tables [10] used to find these boundary points.

\section{The value sets for pairs}

7.1. In 1969 , Aharonov [1] proved that if $\left\{F_{1}, F_{2}\right\}$ is a pair and $\left|z_{1}\right|=r_{1},\left|z_{2}\right|=r_{2}$, $r_{1}, r_{2}<1$, then $\left|F_{1}\left(z_{1}\right) F_{2}\left(z_{2}\right)\right| \leqq r_{1} r_{2}\left(1-r_{1}^{2}\right)^{-1 / 2}\left(1-r_{2}^{2}\right)^{-1 / 2}$, this being sharp if $r_{1}=r_{2}$. In 1972, Jenkins [21] proved that $\left|F_{1}\left(z_{1}\right) F_{2}\left(z_{2}\right)\right| \leqq\left[\mu^{-1}\left(v\left(r_{1}\right)+v\left(r_{2}\right)\right)\right]^{-1}$ where $v(r)$ and $\mu(R)$ are the modules of suitably defined doubly-connected domains. This result is sharp.

In this section we will study the more general problem of finding the boundary of

$$
V\left(r_{1}, r_{2}\right)=\left\{F_{1}\left(r_{1}\right) F_{2}\left(r_{2}\right):\left\{F_{1}, F_{2}\right\} \text { is a univalent pair }\right\},
$$

for any $r_{1}, r_{2}<1$.

From (5.1) we see that if $\left|z_{1}\right|=r_{1},\left|z_{2}\right|=r_{2}$, then $V\left(r_{1}, r_{2}\right)=\left\{F_{1}\left(z_{1}\right) F_{2}\left(z_{2}\right):\left\{F_{1}, F_{2}\right\}\right.$ is a univalent pair\}. Much as in $\S 6.1$ one easily sees that 0,1 are never in any $V\left(r_{1}, r_{2}\right)$ but every other complex number is in one; each $V\left(r_{1}, r_{2}\right) \cup\{0\}$ is compact; $V\left(r_{1}, r_{2}\right)$ is symmetric with respect to reflection in the real axis; and the sets $V\left(r_{1}, r_{2}\right) \cup\{0\}$ are monotone strictly increasing with respect to either variable.

7.2. Again, it is a slight convenience to look at $\log F_{1}\left(r_{1}\right) F_{2}\left(r_{2}\right)$, so we introduce the functional

$$
\Psi\left(F_{1}, F_{2}\right)=\log F_{1}\left(r_{1}\right)+\log F_{2}\left(r_{2}\right) .
$$

Suppose $v$ is any complex number other than 0 or 1 and $v \in \partial V\left(r_{1}, r_{2}\right)$ for some $r_{1}, r_{2}$. It suffices to assume $\operatorname{Im} v \geqq 0$. Let $v=b^{2}$ with $b$ in the first quadrant. Then there must exist a $\lambda$ with $|\lambda|=1$ and a univalent pair $\left\{F_{1}, F_{2}\right\}$ extremal for $\operatorname{Re} \lambda \Psi\left(\mathrm{F}_{1}, F_{2}\right)$ such that $F_{1}\left(r_{1}\right) F_{2}\left(r_{2}\right)=b^{2}$. One easily computes $\lambda A_{1}(w)=$ $=\lambda\left(1-b_{1} b_{2}\right) w /\left(b_{1}-w\right)\left(1-b_{2} w\right)$ and $\lambda A_{2}(w)=\lambda\left(1-b_{1} b_{2}\right) w /\left(b_{2}-w\right)\left(1-b_{1} w\right)$, where $F_{1}\left(r_{1}\right)=b_{1}, F_{2}\left(r_{2}\right)=b_{2}, b_{1} b_{2}=b^{2}$. The pair $\left\{\left(b_{2} / b_{1}\right)^{1 / 2} F_{1},\left(b_{1} / b_{2}\right)^{1 / 2} F_{2}\right\}$ is extremal for the same problem, hence we may assume without loss of generality that $b_{1}=$ $=b_{2}=b$. That is, $F_{1}$ and $F_{2}$ must satisfy

$$
\begin{cases}\frac{\lambda\left(1-b^{2}\right) d w^{2}}{w(b-w)(1-b w)}=\frac{\lambda c_{1}\left(1-r_{1}^{2}\right) d z^{2}}{z\left(r_{1}-z\right)\left(1-r_{1} z\right)} \quad w=F_{1}(z) \\ \frac{\lambda\left(1-b^{2}\right) d w^{2}}{w(b-w)(1-b w)}=\frac{\lambda c_{2}\left(1-r_{2}^{2}\right) d z^{2}}{z\left(r_{2}-z\right)\left(1-r_{2} z\right)} \quad w=F_{2}(z)\end{cases}
$$

where $c_{1}=r_{1} F_{1}^{\prime}\left(r_{1}\right) / F\left(r_{1}\right), c_{2}=r_{2} F_{2}^{\prime}\left(r_{2}\right) / F\left(r_{2}\right)$, and $\lambda c_{1}>0, \lambda c_{2}>0$. 
7.3. The mappings (6.4) and (6.7) were studied in $\S 6.3$ of the last section. The arguments of those paragraphs apply equally well here, and we have the same fundamental periods, $2 \hat{\omega}_{1}, 2 \hat{\omega}_{2}$ of $(6.4), \hat{\omega}_{1}=2 K(b), \hat{\omega}_{2}=2 i K^{\prime}(b)$. Let $B^{2}=\lambda\left(1-b^{2}\right)$, so $B^{2} d \Omega^{2}$ is the quadratic differential on the left-hand side of (7.3).

Suppose $\left\{F_{1}, F_{2}\right\}$ is a univalent pair which satisfies (7.3) for some $r_{1}, r_{2}, \lambda, c_{1}$, $c_{2}$ with $\lambda c_{1}>0, \lambda c_{2}>0, F_{1}\left(r_{1}\right)=b, F_{2}\left(r_{2}\right)=b$. Then $F_{1}$ maps the line segment $\left(0, r_{1}\right)$ onto the unique trajectory of $B^{2} d \Omega^{2}$ which joins 0 to $b$. Since $\lambda$ is the same, $F_{2}$ maps $\left(0, r_{2}\right)$ onto the same trajectory. Therefore, just as in $\S 6.5$, we must have some $\omega_{1}$ satisfying (6.8) such that

$$
\begin{aligned}
{\left[\lambda\left(1-b^{2}\right)\right]^{1 / 2} \omega_{1} } & =\left[\lambda c_{1}\left(1-r_{1}^{2}\right)\right]^{1 / 2} 2 K\left(r_{1}\right) \\
& =\left[\lambda c_{2}\left(1-r_{2}^{2}\right)\right]^{1 / 2} 2 K\left(r_{2}\right)>0 .
\end{aligned}
$$

The boundaries of $F_{1}(U)$ and $F_{2}(U)$ are analytic curves which are also trajectories of $B^{2} d \Omega^{2}$. These need not pass through 1 , but if we assume (as in the conclusion of Theorem 5.1) that these trajectories are mapped into one another by $w \mapsto 1 / w$, then they are mapped by (6.4) onto lines which are parallel to and equidistant from the line through $\pm \omega_{1}+(1 / 2) \omega_{2}$, where $\omega_{2}$ is such that $2 \omega_{1}, 2 \omega_{2}$ is a pair of primitive periods satisfying (6.8). Looking at the composed mappings defined by (7.4), we must thus have $\left[\lambda c_{1}\left(1-r_{1}^{2}\right)\right]^{1 / 2} K^{\prime}\left(r_{1}\right)+\left[\lambda c_{2}\left(1-r_{2}^{2}\right)\right]^{1 / 2} K^{\prime}\left(r_{2}\right)=\operatorname{Im}\left[\lambda\left(1-b^{2}\right)\right]^{1 / 2} \omega_{2}$. Using (7.4), this implies

$$
\operatorname{Im}\left\{\frac{\omega_{2}}{\omega_{1}}\right\}=\frac{1}{2} \frac{K^{\prime}\left(r_{1}\right)}{K\left(r_{1}\right)}+\frac{1}{2} \frac{K^{\prime}\left(r_{2}\right)}{K\left(r_{2}\right)} .
$$

7.4. We now state

Theorem 7.1. Let $b \neq 0,1$ with $0 \leqq \arg b \leqq \pi / 2$ be given. Let $K(b)$ and $K^{\prime}(b)$ be as in the statement of Theorem 6.1. Let $r_{1}, 0<r_{1}<1$ be such that $\operatorname{Re}\left\{K^{\prime}(b) / K(b)\right\}>$ $>(1 / 2) K^{\prime}\left(r_{1}\right) / K\left(r_{1}\right)$. Then there exists a unique $r_{2}$ with $0<r_{2}<1$, $\lambda$ with $|\lambda|=1, c_{1}$ and $c_{2} \neq 0$, and a univalent pair $\left\{F_{1}, F_{2}\right\}$ with $F_{1}\left(r_{1}\right)=F_{2}\left(r_{2}\right)=b$ such that

and

$$
\begin{gathered}
\operatorname{Re}\left\{\frac{K^{\prime}(b)}{K(b)}\right\}=\frac{1}{2} \frac{K^{\prime}\left(r_{1}\right)}{K\left(r_{1}\right)}+\frac{1}{2} \frac{K^{\prime}\left(r_{2}\right)}{K\left(r_{2}\right)}, \\
{\left[\lambda\left(1-b^{2}\right)\right]^{1 / 2} K(b)>0,} \\
\lambda c_{1}>0, \quad \lambda c_{2}>0,
\end{gathered}
$$

$$
\left[\lambda\left(1-b^{2}\right)\right]^{1 / 2} K(b)=\left[\lambda c_{1}\left(1-r_{1}^{2}\right)\right]^{1 / 2} K\left(r_{1}\right)=\left[\lambda c_{2}\left(1-r_{2}^{2}\right)\right]^{1 / 2} K\left(r_{2}\right) .
$$

Further, $F_{1}$ and $F_{2}$ satisfy the differential equations (7.3) with $c_{1}=r_{1} F_{1}^{\prime}\left(r_{1}\right) / F_{1}\left(r_{1}\right)$, $c_{2}=r_{2} F_{2}^{\prime}\left(r_{2}\right) / F_{2}\left(r_{2}\right)$. The complex number $b^{2}$ is on the boundary of $V\left(r_{1}, r_{2}\right)$ and $\left\{F_{1}, F_{2}\right\}$ is the unique univalent pair with $F_{1}\left(r_{1}\right)=F_{2}\left(r_{2}\right)=b$ unless $b$ is real and greater than 1 , in which case there is exactly one more pair, $\left\{\overline{F_{1}(\bar{z})}, \overline{F_{2}(\bar{z})}\right\}$. 
The proof of this theorem is omitted since it follows the proof of Theorem 6.1 quite closely.

7.5. Comparing Theorems 6.1 and 7.1, we easily prove:

Theorem 7.2. Let $\left\{F_{1}, F_{2}\right\}$ be a univalent pair and let $z_{1}, z_{2} \in U$. Then there exists a real $r$ between $r_{1}=\left|z_{1}\right|$ and $r_{2}=\left|z_{2}\right|$ and an $F \in \mathscr{E}$. such that $K^{\prime}(r) / K(r)=$ $=(1 / 2) K^{\prime}\left(r_{1}\right) / K\left(r_{1}\right)+(1 / 2) K^{\prime}\left(r_{2}\right) / K\left(r_{2}\right)$ and $F(r)^{2}=F_{1}\left(z_{1}\right) F_{2}\left(z_{2}\right)$.

Another theorem whose proof is immediate is

Theorem 7.3. Let $0<r<1,\left|z_{1}\right| \leqq r,\left|z_{2}\right| \leqq r$. If $\left\{F_{1}, F_{2}\right\}$ is any univalent pair, then $F_{1}\left(z_{1}\right) F_{2}\left(z_{2}\right) \neq r^{2}$ except when $\left|z_{1}\right|=\left|z_{2}\right|=r, F_{1}(z)=c\left(r / z_{1}\right) z, F_{2}(z)=c^{-1}\left(r / z_{2}\right) z$, and $c \neq 0$ is arbitrary.

Since $\left\{F\left(e^{i \alpha_{1}} z\right), F\left(e^{i \alpha_{2}} z\right)\right\}$ is a univalent pair for any $F \in \mathscr{E}$, Theorem 7.3 generalizes the result of Grunsky [13], that $F\left(z_{1}\right) F\left(z_{2}\right) \neq r^{2}$ for any $F \in \mathscr{E}$ and $z_{1}, z_{2}$ in $U$ with $\left|z_{1}\right|<r,\left|z_{2}\right|<r$.

Maximizing $\left|F_{1}\left(r_{1}\right) F_{2}\left(r_{2}\right)\right|$ is the same as maximizing $\operatorname{Re} \log F_{1}\left(r_{1}\right) F_{2}\left(r_{2}\right)$. The extremal pair must satisfy (7.3) with $\lambda=1$, and hence (6.16) must hold. That is, $b$ is real, $0<b<1$, or is pure imaginary.

In the first case, Theorem 7.1 shows that $\left|F_{1}\left(r_{1}\right) F_{2}\left(r_{2}\right)\right|=r_{1} r_{2}$. However, putting $F_{1}(z)=F_{2}(z)=F_{r_{1}, \pi / 2}(z)$, the Jenkins function of $(6.1)$ gives $F_{1}\left(r_{1}\right) F_{2}\left(r_{2}\right)=$ $=r_{1} r_{2} /\left(1-r_{1} r_{2}\right)>r_{1} r_{2}$. Hence $b$ must be imaginary.

If $b$ is imaginary, say $b=i \beta$, then just as in $\S 6.7,\left|F_{1}\left(r_{1}\right) F_{2}\left(r_{2}\right)\right|=\beta^{2}=r^{2} /\left(1-r^{2}\right)$ where $r$ satisfies $K^{\prime}(r) / K(r)=(1 / 2) K^{\prime}\left(r_{1}\right) / K\left(r_{1}\right)+(1 / 2) K^{\prime}\left(r_{2}\right) / K\left(r_{2}\right)$. This is equivalent to the result of Jenkins mentioned in $\S 7.1$.

\section{The maximum of $\left|b_{2}\right|$ in $\mathscr{E}\left(\left|b_{1}\right|\right)$}

8.1. For any $\beta, 0<\beta<1$, let $\mathscr{E}(\beta)$ denote the set of all $F \in \mathscr{E}$ such that $\left|b_{1}\right|=\beta$, where as in (1.1), $F(z)=b_{1} z+b_{2} z^{2}+\ldots . \mathscr{E}(\beta)$ is compact for each $\beta$. We wish to find the maximum of $\left|b_{2}\right|$ for $F \in \mathscr{E}(\beta)$ for each fixed $\beta$.

Jenkins has solved this problem [20] in the sense that he has implicitly defined (in terms of their mappings) a one parameter family of functions which achieve the maxima. Here we shall duplicate most of his results using variational techniques and carry the analysis further to obtain the actual bounds in a more explicit form. For $\beta<0.827 \ldots$ these bounds are extremely simple.

We may assume $b_{1}>0$ and look for the maximum $\left|b_{2}\right|$ with $b_{1}$ fixed. The extremal $F \in \mathscr{E}\left(b_{1}\right)$ exists and is locally extremal in $\mathscr{E}$ for $\operatorname{Re} \Psi(F)$ where

$$
\Psi(F)=\lambda \log b_{1}+\log b_{2} \text {. }
$$

Here $\lambda$ is a Lagrange multiplier. For this $\Psi, L(F ; G)=\lambda \chi_{1}(G) / b_{1}+\chi_{2}(G) / b_{2}$ where $\chi_{v}(G)$ is the $v$-th coefficient of $G$. Then $L\left(z F^{\prime}\right)=\lambda+2$. Hence from Theorem 4.1, 
$\lambda$ is real. Computing $A(w)$ and $B(w)$ we find that the extremal $F$ will satisfy

$$
\left[(\lambda+1)+\frac{b_{1}^{2}}{b_{2}}\left(w+\frac{1}{w}\right)\right] \frac{d w^{2}}{w^{2}}=\left[(\lambda+2)+\frac{b_{1}}{b_{2}} \frac{1}{z}+\frac{b_{1}}{\bar{b}_{2}} z\right] \frac{d z^{2}}{z^{2}}
$$

for $|z|<1$. Here we have changed the sign and the right-hand side of (8.2) will therefore be negative for $|z|=1$.

Let $\zeta=e^{-i \alpha}$ and consider the slit variation of (4.2). Since $\varepsilon>0$, we find $\operatorname{Re}\left[(\lambda+2)+\left(2 b_{1} / b_{2}\right) \zeta^{-1}\right] \geqq 0$ for each $\zeta$ with $|\zeta|=1$. This implies $\lambda+2 \geqq 2\left|b_{1} / b_{2}\right|>1$ since $\left|b_{2}\right|<2\left|b_{1}\right|$ for any $F \in \mathscr{E}$. That is, $\lambda>-1$.

Make the simple change of variable $\zeta=\left(\left|b_{2}\right| / \bar{b}_{2}\right) z$ and put

$$
2 W=w+\frac{1}{w}, \quad 2 Z=\zeta+\frac{1}{\zeta} .
$$

Then $w=F(z)$ defines $W$ as a univalent function of $Z$, mapping the exterior of the line segment $[-1,1]$ onto the exterior of a continuum $\Gamma$ containing the points $W= \pm 1$, and having no interior (Theorem 4.2). Since $W^{2}-1=(w-1 / w)^{2} / 4, d w^{2} / w^{2}=$ $=d W^{2} /\left(W^{2}-1\right)$ and hence from (8.2.), $W$ and $Z$ satisfy

where

$$
\frac{(1+\sigma W) d W^{2}}{1-W^{2}}=\varrho \frac{(1+\tau Z) d Z^{2}}{1-Z^{2}}
$$

$$
\sigma=\frac{2 b_{1}^{2}}{b_{2}(\lambda+1)}, \quad \tau=\frac{2 b_{1}}{\left|b_{2}\right|(\lambda+2)}, \quad \varrho=\frac{\lambda+2}{\lambda+1} .
$$

We see $\varrho$ and $\tau$ are real, $\varrho>1,0<\tau \leqq 1$. The last since the right-hand side of (8.4) is real and non-negative for $Z \in[-1,1]$. If $\tau<1$, then the right-hand side of (8.4) has a zero at $-1 / \tau$ and hence the continuum $\Gamma$ will not contain the critical point $s=-1 / \sigma$. If $\tau=1$, then $\Gamma$ must contain $s$.

8.2. We now analyze the continuum $\Gamma$ more carefully. We see that $\Gamma$ contains \pm 1 , and may or may not contain $s$. Except for these points it must consist of analytic arcs which are trajectories of $d \Omega^{2}=(1+\sigma W) d W^{2} /\left(1-W^{2}\right)$.

If $\sigma= \pm 1, d \Omega^{2}$ simplifies. Taking $\sigma=1$, for example, $d \Omega^{2}=d W^{2} /(1-W)$. The unique trajectory of this through -1 is the infinite interval $(-\infty,+1)$ of the real axis. Hence $\Gamma$ consists of a segment $[x, 1],-\infty<x \leqq-1$, and $F$ maps $U$ onto $U$ less a slit $[-1, \alpha],-1 \leqq \alpha<0$. Then, just as in the derivation of (4.2), $F /(1-F)^{2}=b_{1} z /(1-z)^{2}$. Comparing coefficients we find $b_{2}=2 b_{1}\left(1-b_{1}\right)$. This is exactly the familiar Pick bound for the second coefficient of a bounded function [27]. As we shall see, this is not the extreme value. The case $\sigma=-1$ is similar and gives the same bound for $\left|b_{2}\right|$. Notice that in these cases $|\sigma|=1, \tau=1$ and hence $b_{1}=1 / \varrho$.

Hence we assume $\sigma \neq \pm 1$. Then $d \Omega^{2}$ has three finite critical points. At \pm 1 , exactly one trajectory leaves. At $s=-1 / \sigma$, exactly three leave at equal angles. 
Thus $\Gamma$ consists of either: $(\tau<1)$ the points \pm 1 and a single analytic arc not passing through $s$, or: $(\tau=1)$ the points $\pm 1, s$, an arc joining -1 to $s$, an arc joining +1 to $s$, and possibly a segment of a third arc leaving $s$.

Suppose $\gamma_{1}$ and $\gamma_{2}$ are two distinct arcs meeting at $s$ and lying on the trajectories of $d \Omega^{2}$. Then $\int d \Omega$ on $\gamma_{1}+\gamma_{2}$ is real and is equal to the integral on $\gamma_{1}^{\prime}+\gamma_{a}+\gamma_{2}^{\prime}$ where $\gamma_{1}^{\prime}$ and $\gamma_{2}^{\prime}$ are subarcs of $\gamma_{1}$ and $\gamma_{2}$, respectively, lying outside a small circle of radius $a$ centered at $s$, and $\gamma_{a}$ is the smaller of the two arcs of this circle joining $\gamma_{1}^{\prime}$ and $\gamma_{2}^{\prime}$. This, and indeed any path, can be altered homotopically in $\mathbf{C}-\{s, 1,-1\}$ without changing the integral of $d \Omega$.

Next we show that if $J$ is the line segment $[-1,1]$, then either $\int d \Omega$ over $J$ is real, or there are two disjoint subintervals of $J$ for which $\int d \Omega$ is real.

First, suppose $\tau<1$. Then either $\Gamma$ is homotopic (in $\mathbf{C}-\{s, 1,-1\}$ ) to $J$, or the three trajectories leaving $s$ must cross $J$. The above assertion therefore holds. On the other hand, if $\tau=1$, then the pair of trajectories from \pm 1 to $s$ make up a single path homotopic to $J$ or else these, together with an arc of the third trajectory from $s$ to some point of $J$, make up two paths homotopic to disjoint segments of $J$. Again the conclusion follows.

If $x_{1}, x_{2} \in J$ and $\sin \theta_{1}=x_{1}, \sin \theta_{2}=x_{2}$, then $\int_{x_{1}}^{x_{2}} d \Omega=\int_{\theta_{1}}^{\theta_{2}}[1+\sigma \sin \theta]^{1 / 2} d \theta$. This is the weighted mean value of complex numbers on a segment of an hyperbola passing through 1 . Two such disjoint integrals could be real only if $\sigma$ were real.

If $\int d \Omega$ is real over $J$, then so is

$$
\int_{-\pi / 2}^{\pi / 2}[1+\sigma \sin \theta]^{1 / 2} d \theta=\int_{0}^{\pi / 2}\left[(1+\sigma \sin \theta)^{1 / 2}+(1-\sigma \sin \theta)^{1 / 2}\right] d \theta .
$$

The square of the last integrand is $2+2\left(1-\sigma^{2} \sin ^{2} \theta\right)^{1 / 2}$. If $\sigma^{2}$ is not real, this integral is the weighted mean of values on an arc lying entirely in one half plane except for the end point $(\theta=0)$ which lies on the real axis. Since the integral is real, we conclude that $\sigma^{2}$ must be real. We have therefore shown that in any case $\sigma$ is either pure real or pure imaginary.

8.3. Suppose $\sigma$ is real. If $|\sigma|<1$, then the segment $J=[-1,1]$ is the only trajectory joining \pm 1 . Hence $\Gamma=J$ and $F(U)=U$. Since $b_{1}>0, F(z)=z$ and $b_{1}=1, b_{2}=0$. This function however does not belong to the class $\mathscr{E}(\beta)$ with $\beta<1$.

If $\sigma$ is real and $|\sigma|>1$, say $\sigma>0$, then $s=-1 / \sigma$ lies on $J$. The only trajectory from 1 is the segment $(s, 1)$ and the only trajectory from -1 is the infinite segment $(-\infty,-1)$. Hence no bounded $\Gamma$ can satisfy the requirements, and this case cannot occur. The case of $\sigma<0$ is similar.

If $\sigma= \pm 1$, we have already seen that $F$ maps $U$ onto $U$ less a radial slit and $\left|b_{2}\right|=2 b_{1}\left(1-b_{1}\right)$.

If $\sigma=i \mu$ is imaginary, the situation is more complicated. If $F(z)$ is extremal, then so is $\overline{F(\bar{z})}$ and hence we may assume $\mu>0$. One trajectory of $d \Omega^{2}=$ $=(1+i \mu W) d W^{2} /\left(1-W^{2}\right)$ is the ray from $s=i / \mu$ to $\infty$ along the imaginary axis. 
Thus $\Gamma$ consists of an analytic arc joining \pm 1 which is homotopic to $J$, or arcs from 1 to $s$, from -1 to $s$, and possibly a segment of the imaginary axis extending upward from $s$. The second case occurs only when $\tau=1$.

8.4. Suppose $\tau<1$. Then from (8.4) the integral of $d \Omega$ around $\Gamma$ in the $W$-plane will equal the integral around $[-1,1]$ in the $Z$-plane. Therefore we must have

$$
q(\mu)=\sqrt{\varrho} p(\tau)
$$

where, since $\Gamma$ is homotopic to $J$,

$$
q(\mu)=\int_{-1}^{1}\left[(1+i \mu W) /\left(1-W^{2}\right)\right]^{1 / 2} d W=\int_{-\pi / 2}^{\pi / 2}[1+i \mu \sin \theta]^{1 / 2} d \theta
$$

$$
=\sqrt{2} \int_{0}^{\pi / 2}\left[\left(1+\mu^{2} \sin ^{2} \theta\right)^{1 / 2}+1\right]^{1 / 2} d \theta \text {. }
$$

Here we took the real part of the integrand, since the integral $q(\mu)$ is real. Similarly,

$$
p(\tau)=\int_{-1}^{1}\left[(1+\tau Z) /\left(1-Z^{2}\right)\right]^{1 / 2} d Z=\int_{0}^{\pi}(1+\tau \cos \theta)^{1 / 2} d \theta .
$$

This is only one relation among three unknowns. However, from the definition, $|\sigma / \tau|=\mu / \tau=\varrho b_{1}$. Hence

$$
\mu=\varrho \tau b_{1} .
$$

A third relation is obtained from (8.4) with the help of the observation that $Z=-1 / \tau$ corresponds to $W=i / \mu$. Thus, the integral of $d \Omega$ in the $Z$-plane along the line segment $l=[-1 / \tau,-1]$ must equal the integral of $d \Omega$ in the $W$-plane along $L$, the image of $l$. Since $l$ lies along an orthogonal trajectory, $L$ must be the line segment from $i / \mu$ to the point $i Y$ at which $\Gamma$ crosses the imaginary axis. This integral is pure imaginary, while the integral along any part of $\Gamma$ is real. Hence

$$
r(\mu)=\sqrt{\varrho} s(\tau)
$$

where

$$
\begin{aligned}
s(\tau) & =\operatorname{Im} \int_{-1 / \tau}^{-1}\left[(1+\tau Z) /\left(1-Z^{2}\right)\right]^{1 / 2} d Z \\
& =\int_{i}^{1 / \tau}\left[\frac{1-\tau t}{t^{2}-1}\right]^{1 / 2} d t
\end{aligned}
$$


and

$$
\begin{aligned}
r(\mu) & =\operatorname{Im} \int_{-1}^{i / \mu} d \Omega=\operatorname{Im} \int_{-1}^{0} d \Omega+\operatorname{Im} \int_{0}^{i / \mu} d \Omega \\
& =\operatorname{Im} \int_{0}^{\pi / 2}[1-i \mu \sin \theta]^{1 / 2} d \theta+\int_{0}^{1}\left[\frac{1-t}{\mu^{2}+t^{2}}\right]^{1 / 2} d t \\
& =\int_{0}^{1}\left[\frac{1-t}{\mu^{2}+t^{2}}\right]^{1 / 2} d t-\frac{1}{\sqrt{2}} \int_{0}^{\pi / 2}\left[\left(1+\mu^{2} \sin ^{2} \theta\right)^{1 / 2}-1\right]^{1 / 2} d \theta .
\end{aligned}
$$

Possible ambiguity of the sign of the last integral can be resolved easily since $r(\mu)$ must be zero when $i / \mu$ is on $\Gamma$.

8.5. One easily verifies that $p^{\prime}(\tau)<0$ and hence $p(\tau)$ is decreasing. We find $p(0)=\pi, p(1)=2 \sqrt{2}$. Actually, $p(\tau)$ is a simple elliptic integral which can be calculated with the help of [8], for example, to be $p(\tau)=2 \sqrt{1+\tau} E^{\prime}(k), k^{2}=(1-\tau) /(1+\tau)$ where $E^{\prime}$ is the associated complete elliptic integral of the second kind.

Similarly, $s^{\prime}(\tau)<0$ and hence $s(\tau)$ is a descreasing function of $\tau$ with $s(0)=\infty$, $s(1)=0$. Again one can compute $s(\tau)=2 \sqrt{1+\tau}[K(k)-E(k)], \quad k^{2}=(1-\tau) /(1+\tau)$.

The first integral of the last line of (8.11) is a decreasing function of $\mu$ which is infinite at 0 and tends toward zero as $\mu \rightarrow \infty$. The second integral is an increasing function, zero at 0 and tending toward $\infty$ as $\mu \rightarrow \infty$. Hence $r(\mu)$ is a decreasing function of $\mu$ which is infinite at $\mu=0$ and which decreases to 0 at some unique $\mu_{0}$. The $\mu_{0}$ for which $r\left(\mu_{0}\right)=0$ was found, with the help of numerical calculations, to be

$$
\mu_{0}=1.1622005 \ldots
$$

The function $q(\mu)$ is clearly increasing. We have $q(0)=\pi, q\left(\mu_{0}\right)=q_{0}=3.3519319 \ldots$, the last value being found with the help of numerical calculations.

8.6. Let $\tau, 0<\tau<1$, be given. From (8.5) and (8.9), $s(\tau) / p(\tau)=r(\mu) / q(\mu)$. The right-hand expression is a decreasing function of $\mu$ and hence this equation determines a unique $\mu$ between 0 and $\mu_{0}$. Then (8.5) or (8.9) determines $\varrho$. (8.8) determines $b_{1}$. Finally, from the definitions of $\varrho, \sigma$, and $\tau$ we find $\left|b_{2}\right|=2 b_{1}(1 / \tau)(1-1 / \varrho)$ and hence $\left|b_{2}\right|$ is determined. That is, each $\tau$ with $0<\tau<1$ determines a unique $b_{1}$ and $\left|b_{2}\right|$.

8.7. Suppose $\tau=1$. Then $\Gamma$ passes through $i / \mu$ and hence we must have $\mu=\mu_{0}$. Then $b_{1}=\mu_{0} / \varrho, \quad\left|b_{2}\right|=2 b_{1}\left(1-b_{1} / \mu_{0}\right)$. When $\tau=1, d \Omega=\varrho(1-Z)^{-1 / 2} d Z$ in the $Z$-plane. This is regular at $Z=-1$, so the boundary slit, $[-1,1]$ in the $Z$-plane has an "open end" at $Z=-1$, i.e., $d \Omega$ changes sign if we change directions at that point. The integral of $d \Omega$ around this slit, starting at this end, will be the same as the integral of $d \Omega$ around $\Gamma$ in the $w$-plane, starting at the "open end" which will be $i / \mu$ or the tip of the slit extending upward along the imaginary axis. That is, $2 q_{0}+2 v=4 \sqrt{2 \varrho}$, where $v \geqq 0$ is the integral of $d \Omega$ from the tip of the slit to $i / \mu$. 
This implies $\varrho \geqq q_{0}^{2} / 8$ with equality only if the extra slit is of zero length. We have therefore proved most of

Theorem 8.1. Let $F(z)=b_{1} z+b_{2} z^{2}+\ldots \in \mathscr{E}$ be locally extremal for $\operatorname{Re}\left\{\lambda \log b_{1}+\log b_{2}\right\}$ with $b_{1}$ and $\lambda$ real, $0<b_{1}<1,-1<\lambda$. Let $\varrho=(\lambda+2) /(\lambda+1)$. Then one of the following three holds:

$$
\begin{array}{ll}
\left|b_{2}\right|=2 b_{1}\left(1-b_{1}\right), & b_{1}=\frac{1}{\varrho} ; \\
\left|b_{2}\right|=2 b_{1}\left(1-\frac{b_{1}}{\mu_{0}}\right), & b_{1}=\frac{\mu_{0}}{\varrho}, \quad \varrho \geqq \frac{q_{0}^{2}}{8} ; \\
\left|b_{2}\right|=2 b_{1} \frac{1}{\tau}\left(1-\frac{b_{1}}{\mu_{1}}\right), & b_{1}=\frac{\mu_{1}}{\varrho}, \quad \mu_{1}=\frac{\mu}{\tau}, \quad \varrho<\frac{q_{0}^{2}}{8} ;
\end{array}
$$

where in (8.15) $\mu, \tau$, and $\varrho$ satisfy (8.5) and (8.9).

Proof. It only remains to show that $\varrho<q_{0}^{2} / 8$ in the case (8.15) when $\tau<1$. Using the formulas of $\S 8.5$ and the well-known formulas for the derivatives of the complete elliptic integrals (see, for example, 710.00-710.05 in [8]), we find

$$
\frac{d}{d \tau}\left(\frac{s(\tau)}{p(\tau)}\right)=\frac{d k}{d \tau}\left(k / k^{\prime 2}\right)\left(K E^{\prime}+K^{\prime} E-K K^{\prime}\right) / E^{\prime 2} .
$$

The derivative of $K E^{\prime}+K^{\prime} E-K K^{\prime}$ is easily found to be zero and since $K^{\prime}(E-K) \rightarrow 0$ as $k \rightarrow 0$, this factor is the constant $\pi / 2$. Hence

$$
\frac{d}{d \tau}\left(\frac{s(\tau)}{p(\tau)}\right)=\frac{\pi k}{2 E^{\prime 2}\left(1-k^{2}\right)}\left(\frac{d k}{d \tau}\right), \quad k^{2}=\frac{1-\tau}{1+\tau} .
$$

Since $d k / d \tau<0$, we conclude that $s(\tau) / p(\tau)$ is a strictly decreasing function of $\tau$. Hence as $\tau$ increases from 0 to 1 , the $\mu$ satisfying $s(\tau) / p(\tau)=r(\mu) / q(\mu)$ increases from 0 to $\mu_{0}$, and $\varrho=q(\mu)^{2} / p(\tau)^{2}$ increases from 1 to $q_{0}^{2} / 8$.

8.8. How does the theorem apply to the problem of finding the maximum of $\left|b_{2}\right|$ in $\mathscr{E}\left(\left|b_{1}\right|\right)$ for a fixed $b_{1}$ ? Figure 2 shows the results of some numerical calculations. The dashed curve is a plot of the parabola of (8.13). If $\varrho \geqq q_{0}^{2} / 8$ in (8.14), then $b_{1} \leqq 8 \mu_{0} / q_{0}^{2}=0.82752416 \ldots$. The arrow points to this value of $b_{1}$ and the portion of the upper curve in Figure 2 to the left of this arrow is the parabola of (8.14). Numerical computations of (8.15) gave $b_{1}$ as a decreasing function of $\tau$ and resulted in the portion of the curve to the right of $8 \mu_{0} / q_{0}^{2}$.

Analytic proofs of the facts that (8.15) determines $b_{1}$ as a decreasing function of $\tau$ and that (8.13) gives $\left|b_{2}\right|$ lying below the values determined by (8.14) and (8.15) can be avoided by using the result of Jenkins in [20]. There he shows the existence of a one-parameter family of functions $F_{\beta} \in \mathscr{E}$ which achieve the maximum $\left|b_{2}\right|$ in the corresponding $\mathscr{E}(\beta)$. Theorem 8.1 must hold for each such $F_{\beta}$. His description of the mappings shows that they belong to our class with $\sigma$ imaginary, and 
hence (8.13) does not hold. They are therefore exactly the mappings giving rise to (8.14) and (8.15). We conclude that for $F \in \mathscr{E},\left|b_{2}\right| \leqq \Phi\left(\left|b_{1}\right|\right)$ where $\Phi\left(\left|b_{1}\right|\right)$ is the function determined by (8.14) if $\left|b_{1}\right| \leqq 8 \mu_{0} / q_{0}^{2}$ and by (8.15) if $\left|b_{1}\right|>8 \mu_{0} / q_{0}^{2}$.

We remark that the extremal functions determined in this way are unique up to the transformation $\overline{F(\bar{z})}$, because of the uniqueness of the solution of the differential equations. This one ambiguity arises because we had to choose $\mu$ positive or negative. Since $\sigma$ is imaginary, the trajectories of $d \Omega$ are symmetric with respect to reflection in the imaginary axis and $-F(-z)=\overline{F(\bar{z})}$.

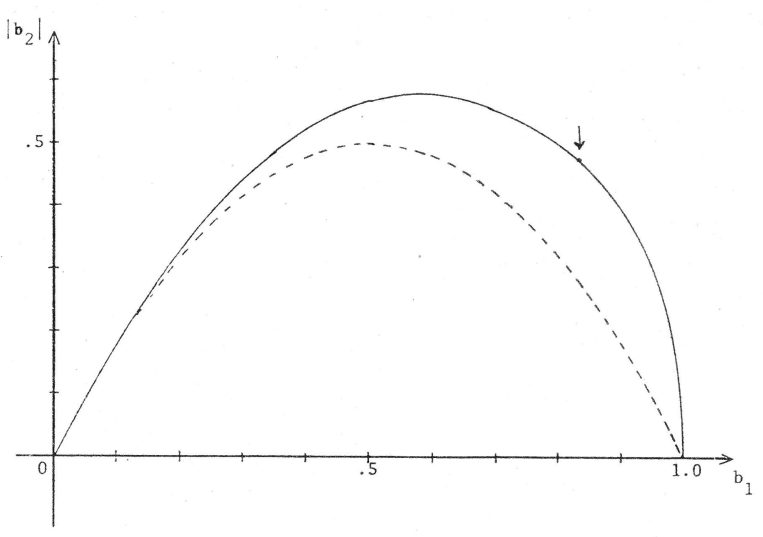

Figure 2

8.9. Let us consider the two special cases when $\lambda=0$ and $\lambda=1$.

Theorem 8.2. Let $F(z)=b_{1} z+b_{2} z^{2}+\ldots \in \mathscr{E}$. Then $\left|b_{2}\right| \leqq \mu_{0} / 2=0.5811002 \ldots$ This is sharp.

Proof. The extremal $F$ must satisfy Theorem 8.1 with $\lambda=0$ and hence $\varrho=2$. If (8.15) were to hold, $\varrho \leqq q_{0}^{2} / 8=1.40443 \ldots$. Hence (8.14) holds with $b_{1}=\left|b_{2}\right|=$ $=\mu_{0} / 2$.

This bound may be compared with the best previously published bound, $\left|b_{2}\right| \leqq$ $\leqq e^{-\gamma / 2}=0.7493 \ldots$, due to Nehari [24] and Aharonov [3].

Theorem 8.3. Let $F(z)=b_{1} z+b_{2} z^{2}+\ldots \in \mathscr{E}$. Then $\left|b_{1} b_{2}\right| \leqq 8 \mu_{0}^{2}=0.4002103 \ldots$. This is sharp.

Proof. Here $\lambda=1$ in (8.1) and $\varrho=3 / 2$. Again (8.15) cannot hold and hence $b_{1}=2 \mu_{0} / 3$. Then $\left|b_{2}\right|=4 \mu_{0} / 9$ and the theorem follows.

Figure 3 shows the image domain $F(U)$ for one of the two extremal functions of Theorem 8.2 (with $b_{1}>0$ ). This was obtained by setting the left-hand side of (8.2) positive and numerically integrating to find the trajectory through 1 . The length of the slit is found as discussed in $\S 8.7$ by computing the integral $v$ required 
to make $q_{0}+v=\sqrt{8 \varrho}$. The function of Theorem 8.3, or for that matter, any of the functions of (8.14), map to the same domain with different slit lengths.

8.10. As a final remark in this section, we observe that we could easily treat the problem of maximizing $\operatorname{Re} b_{2}$ among all $F \in \mathscr{E}$ with a fixed real $b_{1}$. This corresponds to $\Psi(F)=b_{2}+\lambda b_{1}$. Methods similar to those of this section lead to

$$
\frac{2 b_{1}^{2}\left(W-W_{0}\right)}{1-W^{2}} d W^{2}=\frac{2 b_{1}\left(Z-Z_{0}\right)}{1-Z^{2}} d Z^{2}
$$

where $W_{0}=-\left(b_{2}+\lambda b_{1}\right) / 2 b_{1}^{2}, Z_{0}=-\left(2 b_{2}+\lambda b_{1}\right) / 2 b_{1}$ and $Z_{0}$ is real, $Z_{0} \leqq-1$.

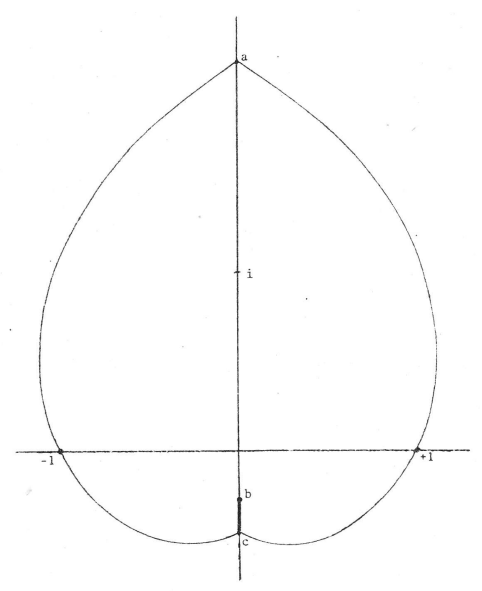

Figure 3

An analysis of the trajectories in this case leads to the conclusion that $W_{0}=-1$ and the extremal functions map $U$ onto $U$ less a radial slit from -1 to $-b_{1} / 4$. That is, if $F \in \mathscr{E}$ and $0<b_{1}<1$, then $\operatorname{Re} b_{2} \leqq 2 b_{1}\left(1-b_{1}\right)$.

\section{The coefficient set of $\left(b_{1}, c_{1}, c_{2}\right)$ for pairs}

9.1. Let $\{F, G\}$ be a univalent pair with $F(z)=b_{1} z+\ldots, G(z)=c_{1} z+c_{2} z^{2}+\ldots$. We wish to determine the set $\mathscr{C}$ of all possible triples $\left(b_{1}, c_{1}, c_{2}\right)$. We can reduce the study of this six dimensional set to that of a two dimensional set.

Let $\mathscr{C}_{1}$ be the set of all $\left(c_{1}, c_{2}\right)$ such that $\{F, G\}$ is a univalent pair with $b_{1}=1$, $c_{1}>0$, and $c_{2}>0$. Given $\left(b_{1}, c_{1}, c_{2}\right) \in \mathscr{C}$, there exists a new pair obtained by the transformations (5.1) so that $\left(1,\left|b_{1} c_{1}\right|,\left|b_{1} c_{2}\right|\right) \in \mathscr{C}$, i.e., so that $\left(\left|b_{1} c_{1}\right|,\left|b_{1} c_{2}\right|\right) \in \mathscr{C}_{1}$. Similarly, if $\mathscr{C}_{1}$ is known, then from (5.1) $\mathscr{C}$ is the set of all $\left(x e^{i \theta_{1}}, x^{-1} y e^{i \theta_{2}}, x^{-1} z e^{i \theta_{3}}\right)$ such that $x>0, \theta_{1}, \theta_{2}, \theta_{3}$ are real and $(y, z) \in \mathscr{C}_{1}$.

For any univalent pair $\left|b_{1} c_{1}\right| \leqq 1$, [1], and $\left|b_{1} c_{2}\right|<2 e^{-\gamma} / \sqrt{3}$, [2], where $\gamma$ is the Euler constant. Further, since $G$ is univalent, $\left|c_{2} / c_{1}\right|<2$. $\mathscr{C}_{1}$ does not contain 
the origin 0 , but we therefore see that $\mathscr{C}_{1} \cup\{0\}$ is compact. We study $\mathscr{C}_{1}$ by fixing $c_{1}$ and finding the maximum $c_{2}$, or, what is equivalent, finding the maximum $\operatorname{Re} b_{1} c_{2}$ for all univalent pairs $\{F, G\}$ with fixed $b_{1} c_{1}>0$ and $b_{1}>0$. If $\{F, G\}$ is extremal for this problem, it will be locally extremal for $\operatorname{Re} \Psi(F, G)$ where

$$
\Psi(F, G)=\lambda b_{1} c_{1}+b_{1} c_{2}
$$

and $\lambda$ is the Lagrange multiplier.

9.2. We apply Theorem 5.1 to (9.1) with $F=F_{1}, G=F_{2}$. We find $L_{1}(H)=$ $=\left(\lambda c_{1}+c_{2}\right) \chi_{1}(H), L_{2}(H)=\lambda b_{1} \chi_{1}(H)+b_{1} \chi_{2}(H)$. Then $A_{1}(w)=-\left[\lambda b_{1} c_{1}+b_{1} c_{2}+b_{1} c_{1}^{2} w\right]$, $A_{2}(w)=-\left[\lambda b_{1} c_{1}+b_{1} c_{2}+b_{1} c_{1}^{2} / w\right], \quad B_{1}(z)=-\left[\lambda b_{1} c_{1}+b_{1} c_{2}\right]$, and $B_{2}(z)=-\left[\lambda b_{1} c_{1}+\right.$ $\left.+2 b_{1} c_{2}+b_{1} c_{1} z+b_{1} c_{1} / z\right]$, where we use the fact that $b_{1} c_{1}, L_{1}\left(z F_{1}^{\prime}\right)=\lambda b_{1} c_{1}+b_{1} c_{2}$, and $L_{2}\left(z F_{2}^{\prime}\right)=\lambda b_{1} c_{1}+2 b_{1} c_{2}$ are all real.

We can assume that the extremal pair have $b_{1}=1, c_{1}>0, c_{2} \geqq 0$. Since $B_{1}(z) \leqq 0$ and $B_{2}(z) \leqq 0$ for $|z|=1, \lambda c_{1}+c_{2} \geqq 0$ and $\lambda c_{1}+2 c_{2} \geqq 2 c_{1}$. Since $c_{2} / c_{1}<2$, this means that $\lambda$ is real and greater than -2 . If $\lambda c_{1}+c_{2}=0$, then $w=F_{1}(z)$ would satisfy. $A(w) d w^{2} / w^{2}=0$, which is impossible. Hence $\lambda c_{1}+c_{2}>0$.

We can therefore write the differential equations of Theorem 5.1 as

where

$$
\begin{gathered}
(1+\alpha w) \frac{d w^{2}}{w^{2}}=\frac{d z^{2}}{z^{2}}, \quad w=F_{1}(z), \\
\left(1+\frac{\alpha}{w}\right) \frac{d w^{2}}{w^{2}}=\varrho\left[1+\frac{\tau}{2}\left(z+\frac{1}{z}\right)\right] \frac{d z^{2}}{z^{2}}, \quad w=F_{2}(z),
\end{gathered}
$$$$
\left\{\begin{array}{lll}
\alpha=\frac{c_{1}^{2}}{\lambda c_{1}+c_{2}}, & \varrho=\frac{\lambda c_{1}+2 c_{2}}{\lambda c_{1}+c_{2}}, & \tau=\frac{2 c_{1}}{\lambda c_{1}+c_{2}}, \\
\alpha>0, & \varrho>1, & 0<\tau \leqq 1, \\
c_{1}=\frac{2 \alpha}{\varrho \tau}, & c_{2}=2 c_{1} \frac{1}{\tau}\left(1-\frac{1}{\varrho}\right), & \lambda=\frac{2(2-\varrho)}{\varrho \tau} .
\end{array}\right.
$$

The last three relations in (9.4) are obtained from the first three by solving for $c_{1}$, $c_{2}$, and $\lambda$.

9.3. We now study the solutions of (9.2) and (9.3). First, (9.2) can be integrated immediately. Using the boundary conditions $w(0)=0, w^{\prime}(0)=b_{1}=1$, we find that $w=F_{1}(z)$ satisfies

$$
2(1+\alpha w)^{1 / 2}+\log \frac{(1+\alpha w)^{1 / 2}-1}{(1+\alpha w)^{1 / 2}+1}=2+\log \frac{\alpha z}{4}, \quad w=F_{1}(z)
$$

Since the right-hand side of (9.2) has no zeros or poles in $U$ except at $0,-1 / \alpha \notin F_{1}(U)$. It may be on the boundary or be exterior; $-\alpha$ will correspondingly be on the boundary or interior to $F_{2}(U)$, and $\tau=1$ or $\tau<1$, respectively.

Suppose $0<\tau<1$. Then $\Gamma_{2}$, the boundary of $F_{2}(U)$ will be an analytic curve, an orthogonal trajectory of the left-hand side of (9.3). Dividing by $2 \pi i$ and integrating 
around the unit circle, we find from (9.3) that

Hence

$$
\frac{\sqrt{\varrho}}{2 \pi} \int_{-\pi}^{\pi}[1+\tau \cos \theta]^{1 / 2} d \theta=\frac{1}{2 \pi i} \int_{\Gamma_{2}}[1+\alpha / w]^{1 / 2} d w=1 .
$$

$$
\sqrt{\varrho} p(\tau)=\pi
$$

where $p(\tau)$ is defined in (8.7). Given $\tau$, this determines a unique $\varrho$ with $1<\varrho<$ $<\pi^{2} / 8$.

Let $0<\sigma<1$ be such that $2-\tau\left(\sigma+\sigma^{-1}\right)=0$. Then the right-hand side of (9.3) has a zero at $-\sigma$. The segment $(-1,-\sigma)$ is a trajectory of this quadratic differential and corresponds to a segment $(-\beta,-\alpha)$ in the $w$-plane. Here $-\beta \in \Gamma_{2}$ and $\beta>0$. Integrating along these trajectories,

$$
\sqrt{\varrho} \int_{-1}^{-\sigma}[1+(\tau / 2)(z+1 / z)]^{1 / 2} d z / z=\int_{-\beta}^{-\alpha}[1+\alpha / w]^{1 / 2} d w / w .
$$

Using the substitution $z+1 / z=-2 t$, the left-hand integral is found to be the negative of $s(\tau)$ of (8.10). The right-hand integral is $2 B+\log (1-B) /(1+B)$ where $B=(1-\alpha / \beta)^{1 / 2}$. This also is negative. Since $-\beta \in \partial F_{2}(U),-1 / \beta \in \partial F_{1}(U)$ and hence from $(9.5),-\sqrt{\varrho} s(\tau)=2+\log \left(-\alpha z_{1} / 4\right)$ where $z_{1}$ is some point with $\left|z_{1}\right|=1$. Since this is real, $z_{1}=-1$ and

$$
\alpha=4 e^{-2} \exp \{-\sqrt{\varrho} s(\tau)\} .
$$

From this $c_{1}$ and $c_{2}$ can be determined.

9.4. If $\tau=1$ so that $-1 / \alpha \in \partial F_{2}(U)$, then the situation is much simpler. In this case $-\alpha \in \partial F_{1}(U)$, and from (9.5),

$$
\alpha=4 e^{-2},
$$

and then from (9.4), $c_{1}$ and $c_{2}$ will be determined as a function of $\varrho$.

In this case (9.6) need not hold. Three orthogonal trajectories of the left-hand side of (9.3) meet at equal angles at $-1 / \alpha$. One lies along the line from this point toward 0 . The boundary of $F_{2}(U)$ will consist of a simple closed curve $\Gamma_{2}$, analytic except at $-1 / \alpha$ plus a slit $\Gamma_{1}$ from $-1 / \alpha$ toward $0 . \Gamma_{1}$ may be of zero length. Integrating around $|z|=1$ we find

$$
\frac{\sqrt{\varrho}}{2 \pi i} \int_{|z|=1}(1+z) d z / z^{1 / 2}=\frac{1}{2 \pi i} \int_{\Gamma_{2}}(1+\alpha w)^{1 / 2} d w / w+\frac{2}{2 \pi i} \int_{\Gamma_{1}}(1+\alpha w)^{1 / 2} d w / w
$$

or $\sqrt{\varrho} p(1) / \pi=1+s$ where $s \geqq 0$. Here all integrals are positive. We start at $z=-1$ in the $z$-plane and at the tip of the slit in the $w$-plane. Thus, if $\tau=1$, we find $\varrho \geqq \pi^{2} / 8$; and hence $c_{1}=8 e^{-2} / \varrho \leqq 64 / \pi^{2} e^{2}$. It follows that if $c_{1}>64 / \pi^{2} e^{2}=0.8775891 \ldots$, then only the $\tau<1$ case occurs.

If $\tau<1$, then $c_{1}=8 \pi^{-2} e^{-2} p(\tau)^{2} \tau^{-1} \exp \{-\pi s(\tau) / p(\tau)\}$. With the help of (8.16), the formula for $p(\tau)$ of $\S 8.5$, and the formulas for the derivatives of the 
elliptic integrals, one easily shows that $(d / d \tau) \log c_{1}=4^{-1} \tau^{-1}(1+\tau)^{-1} E^{\prime-2}\left(\pi^{2}-4 E^{\prime} K^{\prime}\right)$, $k^{2}=(1-\tau) /(1+\tau)$. However, $(d / d k) E K=\left(E+k^{\prime} K\right)\left(E-k^{\prime} K\right) / k k^{\prime 2}$ and $(d / d k)\left(E-k^{\prime} K\right)=$ $=-\left(1-k^{\prime}\right)(E-K) / k k^{\prime}>0$. Hence it follows that $E K$ is an increasing function of $k$ whose minimum at $k=0$ (and hence the minimum of $E^{\prime} K^{\prime}$ ) is $\pi^{2} / 4$. Therefore $c_{1}$ is a strictly decreasing function of $\tau$.

As $\tau \rightarrow 0, k \rightarrow 1, k^{\prime} \rightarrow 0, E \rightarrow 1, E^{\prime} \rightarrow \pi / 2, p(\tau) \rightarrow \pi$. Also $\tau=k^{\prime 2} /\left(2-k^{\prime 2}\right)$. Hence

$$
\begin{aligned}
\lim _{\tau \rightarrow 0} c_{1} & =\frac{8}{\pi^{2} e^{2}} \lim _{\tau \rightarrow 0} p(\tau)^{2}\left(2-k^{\prime 2}\right) \exp \left\{-\left(\pi / E^{\prime}\right)\left(K-E+(2 / \pi) E^{\prime} \log k^{\prime}\right)\right\} \\
& =\frac{16}{e^{2}} \lim \exp \left\{-\left(\pi / E^{\prime}\right)\left(K-\log \left(4 / k^{\prime}\right)-E+\log 4+\log k^{\prime}\left(2 E^{\prime} / \pi\right)-1\right)\right\} .
\end{aligned}
$$

However, $K-\log \left(4 / k^{\prime}\right) \rightarrow 0$ and $\log k^{\prime}\left(2 E^{\prime} / \pi-1\right) \rightarrow 0$ (see 112.01 and 900.07 in [8]). Hence $c_{1} \rightarrow 1$ as $\tau \rightarrow 0$.

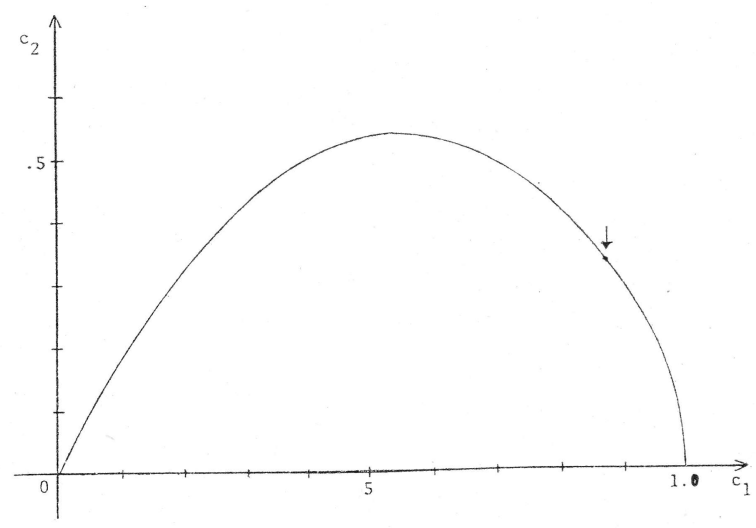

Figure 4

As $\tau \rightarrow 1, s(\tau) / p(\tau) \rightarrow 0$, and $p(\tau) \rightarrow 2 \sqrt{2}$. Therefore $c_{1} \rightarrow 64 / \pi^{2} e^{2}$. Putting these facts together, we have proved:

Theorem 9.1. Let $\left\{F_{1}, F_{2}\right\}$ be a univalent pair with $F_{1}(z)=z+b_{2} z^{2}+\ldots$, $F_{2}(z)=c_{1} z+c_{2} z^{2}+\ldots, c_{1}>0, c_{2}>0$. Then for any $c_{1}, 0<c_{1} \leqq 1$, we have

$$
\begin{aligned}
c_{2} & \leqq 2 c_{1}\left(1-e^{2} c_{1} / 8\right) \quad \text { if } \quad c_{1} \leqq 64 / \pi^{2} e^{2} \\
& \leqq\left(2 c_{1} / \tau\right)(1-1 / \varrho) \quad \text { if } \quad c_{1}>64 / \pi^{2} e^{2}
\end{aligned}
$$

where $\tau$ is the unique real number $0<\tau<1$ such that

$$
c_{1}=\frac{8}{\pi^{2} e^{2}} \frac{p(\tau)^{2}}{\tau} \exp \left\{-\pi \frac{s(\tau)}{p(\tau)}\right\}
$$

and $\varrho=\pi^{2} / p(\tau)^{2}$. The functions $p(\tau)$ and $s(\tau)$ are defined in (8.7) and (8.10). These inequalities are sharp for each $c_{1}$. 
We remark that with the help of the expansion of $E^{\prime}$ near $k^{\prime}=1$ (900.07 in [8]) one can easily show that $c_{2} \rightarrow 0$ as $c_{1} \rightarrow 1$ in (9.9). Some numerical computations were made and Figure 4 shows the bound of (9.9). The arrow points to the "joint" between the regions, i.e., at $c_{1}=64 / \pi^{2} e^{2}=0.8775891 \ldots$ At this point $c_{2}=$ $=128\left(\pi^{2}-8\right) / \pi^{4} e^{2}=0.33248428 \ldots$.

9.5. The pair which maximizes $\left|b_{1} c_{2}\right|$ must be extremal for $\operatorname{Re}\left\{b_{1} c_{2}\right\}$. That is, it will be one of the functions found above with $\lambda=0$ or $\varrho=2$. This belongs to the $\tau=1$ case and the extremal value will be the highest point of the parabolic part of (9.9). That is,

Theorem 9.2. If $\left\{F_{1}, F_{2}\right\}$ is any univalent pair with $F_{1}(z)=b_{1} z+\ldots$ and $F_{2}(z)=c_{1} z+c_{2} z^{2}+\ldots$, then

$$
\left|b_{1} c_{2}\right| \leqq 4 e^{-2} \text {. }
$$

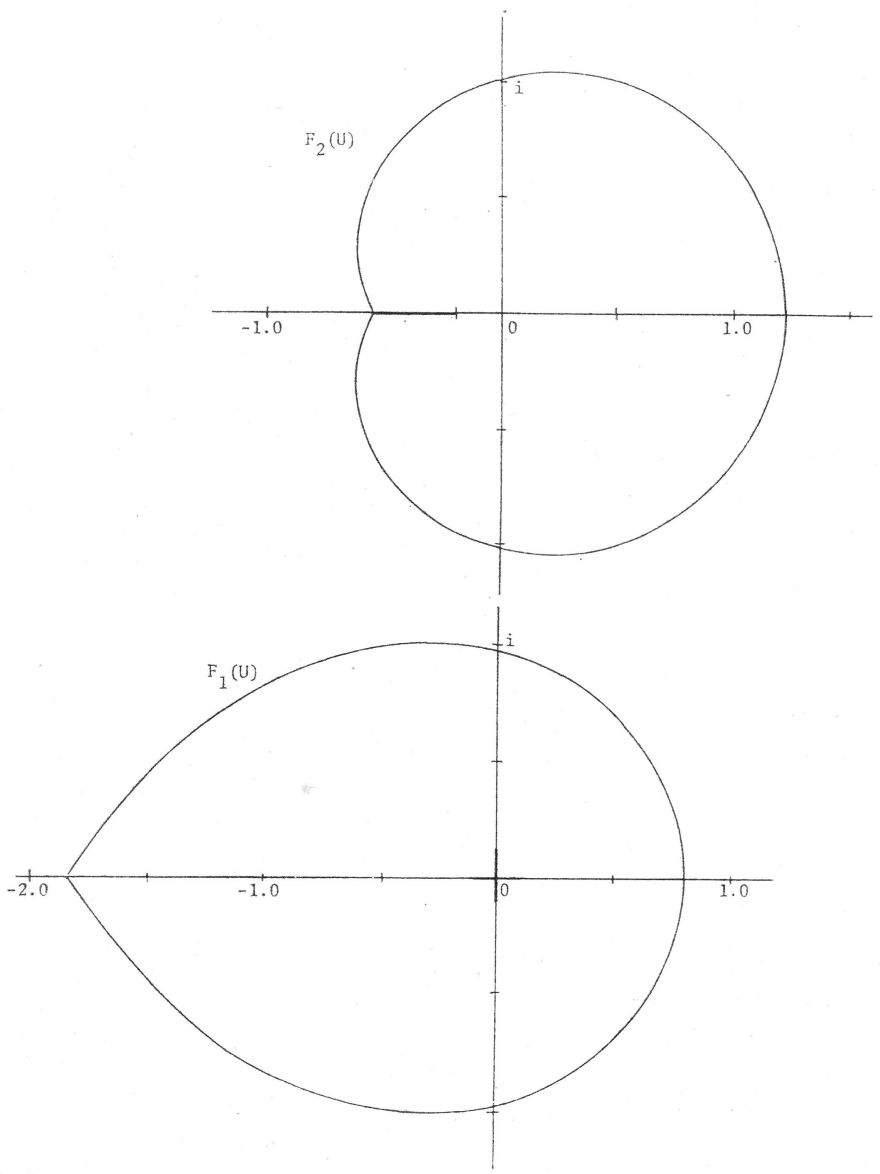

Figure 5 
This result is sharp with the maximum occuring for a pair satisfying (9.2), (9.3) and (9.4) with $\varrho=2, \tau=1$, and $\alpha=4 e^{-2}$. In this case $b_{1} c_{1}=b_{1} c_{2}=4 e^{-2}$.

This bound, $4 e^{-2}=0.5413411 \ldots$, may be compared with Aharonov's bound [2] $2 e^{-\gamma} / \sqrt{3}=0.6483176 \ldots$, or with the bound for Bieberbach-Eilenberg functions $\left|b_{1} b_{2}\right| \leqq 8 \mu_{0}^{2} / 27=0.4002103 \ldots$ (Theorem 8.3).

9.6. Figure 5 shows the typical images of $U$ by the mappings $F_{1}(z)$ and $F_{2}(z)$ which are extremal for Theorem 9.2 with $\tau=1$. The particular pair shown has $F_{1}^{\prime}(0)=b_{1}=1$, hence $-\alpha=-0.5413411 \ldots$ lies on the boundary of $F_{1}(U)$. The boundaries and the length of the slit were obtained by numerical integration.

All extremal pairs of Theorem 9.1 in the case $\tau=1\left(c_{1} \leqq 64 / \pi^{2} e^{2}\right)$ will map onto similar domains but with slits of different lengths.

\section{An inequality of Golusin type}

10.1. The methods of this paper can be used to give a simple proof of an inequality of Golusin type for Bieberbach-Eilenberg functions. This is closely related to inequalities of Grunsky type proved by Jenkins [20] and Garabedian and Schiffer [11], and to the inequalities proved by Nehari in [24]. In fact, it is shown in [17] that inequalities of Golusin and Grunsky type are essentially equivalent. We include the following theorem, however, since it illustrates the reduction which occurs when the $A(w)$ in Theorem 4.1 is a perfect square

Theorem 10.1. Let $F(z) \in \mathscr{E}$. Let $z_{1}, z_{2}, \ldots, z_{N}$ be distinct points of $U$ and let $x_{1}, x_{2}, \ldots, x_{N}$ be complex constants such that

$$
\operatorname{Im} \sum_{v=0}^{\infty} x_{v}=0
$$

Then

$$
\operatorname{Re} \sum_{v=1}^{N} \sum_{\mu=1}^{N} x_{v} x_{\mu} \log \frac{F\left(z_{v}\right)-F\left(z_{\mu}\right)}{\left(z_{v}-z_{\mu}\right)\left[1-F\left(z_{v}\right) F\left(z_{\mu}\right)\right]} \leqq-\sum_{\nu=1}^{N} \sum_{\mu=1}^{N} \bar{x}_{v} x_{\mu} \log \left(1-\bar{z}_{v} z_{\mu}\right) \text {. }
$$

Remarks. Ambiguities in the choice of branches of the logarithms disappear because of (10.1). The requirement that the $z_{v}$ be distinct can be relaxed by taking appropriate limits. When $\mu=v$ in the left-hand sum of $(10.2)$, we let $\left[F\left(z_{v}\right)-F\left(z_{\mu}\right)\right] /\left(z_{v}-z_{\mu}\right)=$ $=F^{\prime}\left(z_{v}\right)$. The right-hand side of (10.2) is always real.

Proof. We apply Theorem 4.1 with

$$
\Psi(F)=\sum_{v=1}^{N} \sum_{\mu=1}^{N} x_{v} x_{\mu} \log \frac{F_{v}-F_{\mu}}{\left(z_{v}-z_{\mu}\right)\left[1-F_{v} F_{\mu}\right]}
$$


where we denote $F\left(z_{v}\right)=F_{v}$. We find with some computation that

$$
\begin{aligned}
A(w) & =-w^{2} \sum_{v=1}^{N} \sum_{\mu=1}^{N} \frac{x_{v} x_{\mu}\left(1-F_{v}^{2}\right)\left(1-F_{\mu}^{2}\right)}{\left(F_{v}-w\right)\left(F_{\mu}-w\right)\left(1-F_{v} w\right)\left(1-F_{\mu} w\right)} \\
& =-w^{2}\left[\sum_{v=1}^{N} x_{v} \frac{\left(1-F_{v}\right)^{2}}{\left(F_{v}-w\right)\left(1-F_{v} w\right)}\right]^{2} .
\end{aligned}
$$

The extremal $F$ maps $U$ onto the interior of an analytic curve $\Gamma$ through \pm 1 satisfying $A(w) d w^{2} / w^{2}>0$. In this case we can take the square root and integrate. We find that for $w \in \Gamma$

$$
\operatorname{Re} \sum_{v=1}^{N} x_{v} \log \frac{1-F_{v} w}{F_{v}-w}=\text { constant. }
$$

Since $w=-1$ is on $\Gamma$, this constant is zero and

$$
\operatorname{Re} \sum_{v=1}^{N} x_{v} \log \frac{F\left(z_{v}\right)-F(z)}{1-F\left(z_{v}\right) F(z)}=0 \quad \text { if } \quad|z|=1 .
$$

Then, for $|z|=1$ we have

$$
\begin{aligned}
\operatorname{Re} \sum_{v=1}^{N} x_{v} \log \frac{F\left(z_{v}\right)-F(z)}{\left(z_{v}-z\right)\left[1-F\left(z_{v}\right) F(z)\right]} & =-\operatorname{Re} \sum_{v=1}^{N} x_{v} \log \left(z_{v}-z\right) \\
& =-\operatorname{Re} \sum_{v=1}^{N} \bar{x}_{v} \log \left(\bar{z}_{v}-\frac{1}{z}\right) \\
& =-\operatorname{Re} \sum_{v=1}^{N} \bar{x}_{v} \log \left(1-\bar{z}_{v} z\right)
\end{aligned}
$$

since $\sum \bar{x}_{v} \log (-1 / z)$ is pure imaginary for $|z|=1$ because of (10.1).

Both sides of (10.3) are the real parts of functions which are analytic in the closure of $U$. Hence equality holds in all of $U$ and

$$
\sum_{v=1}^{N} x_{v} \log \frac{F\left(z_{v}\right)-F(z)}{\left(z_{v}-z\right)\left[1-F\left(z_{v}\right) F(z)\right]}=-\sum_{v=1}^{N} \bar{x}_{v} \log \left(1-\bar{z}_{v} z\right)+i k
$$

where $k$ is some real constant.

Set $z=z_{\mu}$ in (10.4), multiply by $x_{\mu}$, and add the resulting relations for all $\mu$. Then $i \sum x_{\mu} k$ is pure imaginary and hence

$$
\operatorname{Re} \sum_{v=1}^{N} \sum_{\mu=1}^{N} x_{v} x_{\mu} \log \frac{F\left(z_{v}\right)-F\left(z_{\mu}\right)}{\left(z_{v}-z_{\mu}\right)\left[1-F\left(z_{v}\right) F\left(z_{\mu}\right)\right]}=-\sum_{v=1}^{N} \sum_{\mu=1}^{N} \bar{x}_{v} x_{\mu} \log \left(1-\bar{z}_{v} z_{\mu}\right) .
$$

Thus, the extremal function gives equality in (10.2) and the inequality must hold for all $F \in \mathscr{E}$. 


\section{References}

[1] Aharonov, D.: A generalization of a theorem of J. A. Jenkins.-Math. Z. 110, 1969, 218-222.

[2] Aharonov, D.: On pairs of functions and related classes.-Duke Math. J. 40, 1973, 669-676.

[3] Aharonov, D.: On Bieberbach-Eilenberg functions.-Bull. Amer. Math. Soc. 76, 1970, $101-104$.

[4] Ahlfors, L. V.: Conformal invariants. - McGraw-Hill, New York, 1973.

[5] Alenicyn, Yu. E.: A contribution to the theory of univalent and Bieberbach-Eilenberg functions.-Dokl. Akad. Nauk SSSR (N.S) 109, 1956, 247-249. (Russian).

[6] Alenicyn, Yu. E.: On functions without common values and the outer boundary of the domain of values of a function.- Ibid. 115, 1957, 1055-1057 (Russian).

[7] Bieberbach, L.: Über einige Extremalprobleme im Gebiet der konformen Abbildung. - Math. Ann. 77, 1916, 153-172.

[8] Byrd, P. F., and Friedman, M. D.: Handbook of elliptic integrals for engineers and scientists, 2nd ed.-Springer-Verlag, New York-Heidelberg, 1971.

[9] Eilenberg, S.: Sur quelques proprietés topologiques de la surface de sphère. - Fund. Math. $25,1935,267-272$.

[10] Fettis, H. E., and J. C. CAslin: A table of complete elliptic integrals of the first kind for complex values of the modulus, Part I. - Applied Math. Research Lab., Aerospace Research Laboratories, USAF, Wright-Patterson Air Force Base, Ohio (Project No. 7071, Nov. 1969).

[11] Garabedian, P. R., and M. Schiffer: The local maximum theorem for the coefficients of univalent functions. - Arch. Rational Mech. Anal. 26, 1967, 1-32.

[12] Golusin, G. M.: Geometric theory of functions of a complex variable. - Transl. Math. Monographs, vol. 26, Amer. Math. Soc., Providence, R. I., 1969.

[13] Grunsky, H.: Einige Analoge zum Schwarzschen Lemma. - Math. Ann. 108, 1933, 190 - 196.

[14] Guelfer, S.: On the class of regular functions which do not take on any pair of values $w$ and - w. - Mat. Sb. (N. S.) 19 (61), 1946, 33-46 (Russian).

[15] Gunning, R. C.: Lectures on modular forms. - Ann. of Math. Studies, no. 48, Princeton University Press, Princeton, N. J., 1962.

[16] Hummel, J. A.: Inequalities of Grunsky type for Aharonov pairs. - J. Analyse Math. 25, $1972,217-257$.

[17] Hummel, J. A., and M. Schiffer: Coefficient inequalities for Bieberbach-Eilenberg functions. - Arch. Rational Mech. Anal. 32, 1969, 87-99.

[18] Jenkins, J. A.: On Bieberbach-Eilenberg functions. - Trans. Amer. Math. Soc. 76, 1954, 389-396.

[19] Jenkins, J. A.: On Bieberbach-Eilenberg functions. II. - Ibid. 78, 1955, 510-515.

[20] Jenkins, J. A.: On Bieberbach-Eilenberg functions. III. - Ibid. 119, 1965, 195-215.

[21] Jenkins, J. A.: A remark on "pairs" of functions. - Proc. Amer. Math. Soc. 31, 1972, 119121.

[22] Lebedev, N. A.: An Application of the area principle to non-overlapping domains. - Trudy Mat. Inst. Stekloy. 60, 1961, 211-231 (Russian).

[23] Lebedev, N. A., and I. M. Milin: On the coefficients of certain classes of analytic functions. - Mat. Sb. (N. S.) 28(70), 1951, 359-400 (Russian).

[24] Nehari, Z.: Some inequalities in the theory of functions. - Trans. Amer. Math. Soc. 75, 1953, 256-286.

[25] Nehari, Z.: On the coefficients of Bieberbach-Eilenberg functions. - J. Analyse Math. 23, $1970,297-303$.

[26] Nelson, D. J.: Extremal problems in the class of Bieberbach-Eilenberg functions. - Dissertation, Stanford University, September 1972. 
[27] PICK, G.: Über die konforme Abbildung eines Kreises auf ein schlichtes und zugleich beschränktes Gebiet. - Sitzungsber. Math. - Natur. Kaiserlichen Akad. der Wissensch. Wien, 126, 1917, 247-263.

[28] Rogosinski, W.: On a theorem of Bieberbach-Eilenberg. - J. London Math. Soc. 14, 1939, $4-11$.

[29] Rogosinski, W.: On the coefficients of subordinate functions. - Proc. London Math. Soc. (2) $48,1943,48-82$.

[30] Schaeffer, A. C., M. Schiffer, and D. C. Spencer: The coefficient regions of schlicht functions. - Duke Math. J. 16, 1949, 493-526.

[31] Schiffer, M.: A method of variations within the family of simple functions, - Proc. London Math. Soc. (2) 44, 1938, 432--449.

[32] Schiffer, M.: Extremum problems and variational methods in conformal mapping. - Proc. International Congress Math., Edinburgh, 1958, 213-231.

[33] SCHIfFer, M.: Inequalities in the theory of univalent functions. - Inequalities-III, Academic Press, New York-London, 1972, 311-319.

University of Maryland

Department of Mathematics

College Park, Maryland 20742

USA

Received 11 September 1975
Stanford University

Department of Mathematics

Stanford, California 94305

USA 